\title{
Die kinetische Energie ionisierter Molekülfragmente
}

\author{
VI. Einfach- und doppeltgeladene Ionen hoher Anfangsenergie \\ in den Bruchstückspektren von Kohlenwasserstoffen

\section{R. FUCHS}

Mitteilung aus der Physikalisch-Technischen Bundesanstalt, Braunschweig

(Z. Naturforschg. 21 a, 2069—2082 [1966] : eingegangen am 24. September 1966)

\begin{abstract}
It is well known that in the initial energy spectra of hydrocarbon fragment ions formed by electron impact, satellite ion groups occur which are believed to be mainly due to the fragmentation of doubly charged ions into two singly charged fragments. By using an electron energy of $150 \mathrm{eV}$ a new type of satellite was observed in the initial energy spectra of $\mathrm{C}_{1} \mathrm{H}_{k}{ }^{+}$and $\mathrm{C}_{2} \mathrm{H}_{l}^{+}$fragment ions from hydrocarbons with three and more carbon atoms. The $n$-paraffins were investigated systematically up to n-decane. Because the initial energy of these satellites is nearly twice the initial energy of the already well known "first" satellites, it is suggested that these newly discovered "second" satellites are due to an analogous fragmentation process of the type
\end{abstract}

$$
\mathrm{A}^{+++} \rightarrow \mathrm{B}^{+}+\mathrm{C}^{++} \text {. }
$$

Therefore, a careful search was made for the corresponding doubly charged fragment ions of high kinetic energy. Well pronounced satellite groups of doubly charged fragment ions were, indeed, found. Their momentum approximately equals the momentum of the corresponding "second" satellites of singly charged fragments, which supports the suggested mechanism.

In einer der früheren Arbeiten (IV) ${ }^{1}$, in der über Ionen hoher Anfangsenergie (AE) berichtet worden war, wurde darauf hingewiesen, da $\beta$ in den Anfangsenergieverteilungen der $\mathrm{C}_{1} \mathrm{H}_{k}{ }^{+}$-Ionen $\left(\mathrm{C}_{1^{-}}\right.$Gruppe $^{2}$ ) von $\mathrm{n}$-Hexan neben den „normalen“ 1. Satellitgruppen jeweils eine weitere Gruppe ${ }^{2}$ mit höherer Anfangsenergie - in IV x-Satellit genannt auftritt. Während die Entstehung der 1. Satellitgruppe mit großer Wahrscheinlichkeit dem Zerfall eines doppeltgeladenen Molekülions in zwei einfachgeladene Bruchstücke zugeschrieben werden kann (s. Anm. 1, 3-7), war es dort noch nicht möglich, eine Deutung für den Ursprung dieser 2. Gruppe zu geben.

An Hexan waren die Messungen jedoch gegenüber den anderen Substanzen mit einer etwas geänderten Ionenquelle ausgeführt worden, bei der für die effektive Elektronenenergie neben der Spannung zwischen Kathode und Ionisierungskasten zusätzlich noch die Hälfte der Ziehspannung maßgebend ist. Bei einer Ziehspannung von $100 \mathrm{~V}$ betrug die Elek-

1 R. Fuchs u. R. Taubert, Z. Naturforschg. 19 a, 1181 [1964].

2 Über die Bedeutung dieser und anderer Bezeichnungen siehe auch I, R. TAUBERT, Z. Naturforschg. 19 a, 484 [1964] und II, R. Fuchs u. R. Taubert, Z. Naturforschg. 19 a, 494 [1964]. Zur Unterscheidung von einfach- und doppeltgeladenen Ionen sollen in dieser Arbeit die Bezeichnungen "C $\mathrm{C}_{\mathrm{i}}^{+}$-Gruppe“ bzw. „ $\mathrm{C}_{\mathrm{i}}^{++}$-Gruppe“ gebraucht werden. Die Satelliten in den Verteilungen sollen hier durch ihre Numerierung mit zunehmender Energie, also durch 1., 2., .... Satellitgruppe, gekennzeichnet werden, wobei die sogen. sekundären Satelliten (siehe IV) unberücksichtigt bleiben. tronenenergie somit nicht $70 \mathrm{eV}$, sondern rund 120 $\mathrm{eV}$.

Es wurde deshalb vermutet, daß auch bei anderen Kohlenwasserstoffen in den Anfangsenergieverteilungen der $\mathrm{C}_{1}{ }^{+}$-Gruppen solche 2 . Satelliten bei einer Erhöhung der Elektronenenergie auftreten. Wenn aber solche 2. Satelliten in der $\mathrm{C}_{1}{ }^{+}$-Gruppe nicht nur vereinzelt gefunden werden, sondern bei erhöhter Elektronenenergie in ähnlicher Weise eine allgemeine Erscheinung darstellen, wie die „normalen" 1. Satelliten, so kann man erwarten, daß auch in den anderen C-Gruppen solche 2. Satelliten auftreten. Wie früher wird es dann für das Auffinden von systematischen Gesetzmäßigkeiten zweckmäßig sein, eine größere Anzahl von Kohlenwasserstoffen in die Messungen mit einzubeziehen. In diesem $\mathrm{Zu}$ sammenhang ist es interessant, bei diesen Substanzen auch unter den doppeltgeladenen Bruchstücken nach Ionen hoher Anfangsenergie, d. h. nach Satelliten zu suchen, und zwar dort, wo die Kohlenstoffkette gegenüber dem Molekül um mindestens ein

3 P. Kusch, A. Hustrulid u. J. T. Tate, Phys. Rev. 52, 843 [1937]. - A. Hustrulid, P. Kusch u. J. T. Tate, Phys. Rev. 54, 1037 [1938].

4 C. E. Berry, Phys. Rev. 78, 597 [1950].

5 H. E. Stanton, J. Chem. Phys. 30, 1116 [1959].

6 T. Tsuchiya, J. Chem. Phys. 36, 568 [1962].

7 J. Bracher, H. Ehrhardt, R. Fuchs, O. Osberghaus u. R. T AUbert, Advances in Mass Spectrometry II, herausg. von R. M. Elliott, Pergamon Press, London 1963, S. 285 ff. 
C-Atom verkürzt ist ${ }^{8}$. Für solche Messungen dürfte sich unter anderem besonders gut die homologe Reihe der n-Paraffine eignen, wo man schon auf Grund der von Glied zu Glied sich gleichmäßig verlängernden Kohlenstoffkette eine Systematik erwarten darf.

\section{Experimentelles}

Die Apparatur war im wesentlichen die gleiche wie die zu den früheren Messungen (I, II) benutzte, nur die Ionenquelle war im Hinblick auf eine direkte Kalibrierung des Ablenkkondensators über die thermische Energieverteilung von Molekülionen etwas anders konstruiert: Die Ziehelektrode war unmittelbar mit dem Ionisierungskasten verbunden und der
Ionenrepeller war mit einer Zusatzheizung versehen. Auf diese Weise konnte die gesamte Wandung des Ionisierungsraumes auf eine einheitliche Temperatur - meßbar durch 2 Thermoelemente - gebracht werden. Für das auch hier als interner Standard dienende Satellitmaximum der $\mathrm{CH}_{3}{ }^{+}$-Ionen von Propan ergab sich damit eine Anfangsenergie von 2,71 $\pm 0,05 \mathrm{eV}$. Diese liegt also um rund $8 \%$ höher als der auf andere Weise ermittelte Wert 2,5 eV, auf dem die in II mitgeteilten Anfangsenergien basieren. Bei einem Vergleich der jetzigen Ergebnisse mit den früheren ist dies zu berücksichtigen.

Der Ionenrepeller hatte von der Ziehplatte einen Abstand von $4 \mathrm{~mm}$, der „Elektronenteppich“ lag in der Mitte zwischen beiden Elektroden. Die in den Ionisierungsraum eintretenden Elektronen gewinnen

\begin{tabular}{|c|c|c|c|c|c|c|c|c|c|c|c|c|c|c|c|}
\hline \multicolumn{7}{|c|}{ Anfangsenergie } & & & & & & & & & \\
\hline \multirow{2}{*}{$M$} & \multirow{2}{*}{ Ion } & \multicolumn{2}{|c|}{ Propan } & \multicolumn{2}{|c|}{ n-Butan } & \multicolumn{2}{|c|}{ i-Butan } & \multicolumn{2}{|c|}{ n-Pentan } & \multicolumn{2}{|c|}{ i-Pentan } & \multicolumn{2}{|c|}{ neo-Pentan } & \multicolumn{2}{|c|}{ n-Hexan } \\
\hline & & 1.S & $2 . \mathrm{S}$ & $1 . S$ & $2 . \mathrm{S}$ & $1 . \mathrm{S}$ & $2 . \mathrm{S}$ & $1 . \mathrm{S}$ & $2 . \mathrm{S}$ & $1 . \mathrm{S}$ & $2 . \mathrm{S}$ & $1 . \mathrm{S}$ & $2 . \mathrm{S}$ & $1 . \mathrm{S}$ & $2 . \mathrm{S}$ \\
\hline $\begin{array}{l}14 \\
15\end{array}$ & $\begin{array}{l}\mathrm{CH}_{2}{ }^{+} \\
\mathrm{CH}_{3}{ }^{+}\end{array}$ & $\begin{array}{l}\text { a } \mathbf{2 , 8 6} \\
\text { a } 2,71\end{array}$ & $(\bar{c} \overline{5,49})$ & $\begin{array}{l}\text { a } \mathbf{2 , 6 8} \\
\text { a } \mathbf{2 , 5 2}\end{array}$ & $\begin{array}{l}\text { c } 5,51 \\
\text { b } 5,68\end{array}$ & $\begin{array}{l}\text { a } \mathbf{2 , 9 2} \\
\text { a } \mathbf{2 , 6 9}\end{array}$ & \begin{tabular}{|l|} 
c 5,63 \\
b 5,96
\end{tabular} & a $\mathbf{2 , 3 0}$ & b 5,31 & a 2,55 & b 5,47 & $\begin{array}{l}\text { a } \mathbf{2 , 9 3} \\
\text { a } \mathbf{2 , 6 2}\end{array}$ & $\begin{array}{l}\text { c } 5,86 \\
\text { b 5,91 }\end{array}$ & a 2,19 & b 5,06 \\
\hline $\begin{array}{l}25 \\
26 \\
27 \\
28\end{array}$ & $\begin{array}{l}\mathrm{C}_{2} \mathrm{H}^{+} \\
\mathrm{C}_{2} \mathrm{H}_{2}+ \\
\mathrm{C}_{2} \mathrm{H}_{3}^{+} \\
\mathrm{C}_{2} \mathrm{H}_{4}^{+} \\
\\
\mathrm{C}_{2} \mathrm{H}_{5}^{+}\end{array}$ & $\begin{array}{l}\text { a } 1,30 \\
\text { a } 1,52\end{array}$ & & $\begin{array}{l}\text { b } 2,04 \\
\text { a } 2,13 \\
\text { b } 2,02 \\
\text { (c0,75s) } \\
\text { a } 2,21\end{array}$ & $\begin{array}{l}- \\
- \\
-\end{array}$ & b 2,09 & & \begin{tabular}{|l} 
a 2,12 \\
a 2,17 \\
(c 2,10$)$ \\
b $1,02 \mathrm{~s}$ \\
a 2,06
\end{tabular} & $\begin{array}{c}- \\
\overline{-} \\
(c 4,05) \\
(c 4,44)\end{array}$ & $\begin{array}{l}\text { b } 2,24 \\
\text { a 2,26 } \\
\text { b } 2,37 \\
\text { b } 2,39 \\
\text { b } 0,98 \mathrm{~s} \\
\text { a } 2,19\end{array}$ & $\left(\begin{array}{c}-\bar{c} 4,13) \\
\mathrm{c} 4,57\end{array}\right.$ & $\begin{array}{l}\text { b } \mathbf{2 , 4 5} \\
\text { b } 2,28\end{array}$ & & $\begin{array}{l}\text { b } 2,04 \\
\text { b 2,14 } \\
\text { b 2,03 } \\
\text { b 2,12 } \\
\text { b } 0,62 \mathrm{~s} \\
\text { a } 1,90\end{array}$ & $\begin{array}{c}- \\
\mathrm{c} 4,28 \\
\mathrm{c} 4,18 \\
\mathrm{c4,38}\end{array}$ \\
\hline $\begin{array}{l}37 \\
38 \\
39 \\
40\end{array}$ & $\begin{array}{l}\mathrm{C}_{3} \mathrm{H}^{+} \\
\mathrm{C}_{3} \mathrm{H}_{2}+ \\
\mathrm{C}_{3} \mathrm{H}_{3}+ \\
\mathrm{C}_{3} \mathrm{H}_{4}+ \\
\\
\mathrm{C}_{3} \mathrm{H}_{5}{ }^{+} \\
\mathrm{C}_{3} \mathrm{H}_{6} \\
\\
\mathrm{C}_{3} \mathrm{H}_{7}+\end{array}$ & & & b 0,97 & & c 1,00 & & $\begin{array}{l}\text { b } 1,42 \\
\text { b } 1,46 \\
\text { a } 1,47\end{array}$ & & $\begin{array}{l}\text { c } 1,56 \\
\text { c } 1,57\end{array}$ & & & & $\begin{array}{l}\text { b } 1,71 \\
\text { b } 1,70 \\
\text { c } 1,36 \\
\text { c } 1,62 \\
\text { c } 1,64 \\
\text { c } 0,87 \text { s } \\
\text { a } 1,77\end{array}$ & $\begin{array}{c}- \\
- \\
- \\
(\mathrm{c} 3,95) \\
-\end{array}$ \\
\hline $\begin{array}{l}50 \\
51 \\
52 \\
53 \\
54 \\
55 \\
56 \\
57\end{array}$ & $\begin{array}{l}\mathrm{C}_{4} \mathrm{H}_{2}{ }_{4} \\
\mathrm{C}_{4} \mathrm{H}_{3}^{+} \\
\mathrm{C}_{4} \mathrm{H}_{4}^{+} \\
\mathrm{C}_{4} \mathrm{H}_{5}^{+} \\
\mathrm{C}_{4} \mathrm{H}_{6} \\
\mathrm{C}_{4} \mathrm{H}_{7}^{+} \\
\mathrm{C}_{4} \mathrm{H}_{8^{+}} \\
\mathrm{C}_{4} \mathrm{H}_{9^{+}}\end{array}$ & & & & & & & $\begin{array}{l}\text { b } \mathbf{0 , 6 4} \\
\text { c } 0,77\end{array}$ & & $\begin{array}{l}\text { c } 0,74 \\
\text { b } 0,71 \\
\text { c } 0,75 \\
\text { c } 0,76\end{array}$ & & $\begin{array}{l}\text { c } 0,78 \\
\text { c } 0,77\end{array}$ & & $\begin{array}{l}\text { b } \mathbf{0 , 9 6} \\
\text { c 1,03 } \\
\text { b } 1,10\end{array}$ & \\
\hline
\end{tabular}

Tab. 1. Anfangsenergien der 1. und 2. Satellitgruppen (1.S und 2.S) von einfachgeladenen Ionen, gemessen an den Stellen intensität der betreffenden Linie aus. Eingeklammerte Werte gehören zu nicht aufgelösten Satelliten und sind unsicher. Die deutungen einer Satellitgruppe. Kein Zeichen bedeutet: Die Verteilung des betreffenden Ions wurde nicht untersucht. Die Satellitmaximum durch ein oder zwei Minima von der übrigen Verteilung getrennt, die im Falle a niedriger sind als die halbe Satellit (siehe IV). a) Die Deutung für die

\footnotetext{
${ }^{8}$ Die in II erwähnten nur quasithermischen AE-Verteilungen von doppeltgeladenen Ionen bezogen sich auf solche Bruchstücke, die sich vom Molekülion nur durch das Fehlen von einem oder mehreren H-Atomen unterscheiden.
} 
also zusätzlich eine etwa der halben Ziehspannung entsprechende Energie. Die Elektronenbeschleunigungsspannung und die Ziehspannung betrugen bei der vorliegenden Arbeit beide $100 \mathrm{~V}$, so daß sich für die effektive Elektronenenergie ca. $150 \mathrm{eV}$ ergaben.

Die Nachweisempfindlichkeit konnte durch einen 9-stufigen Sekundärelektronenvervielfacher mit $\mathrm{Cu}$ Be-Dynoden ${ }^{9}$ so gesteigert werden, daß trotz einer Verringerung des Druckes in der Ionenquelle gegenüber früher (II) um einen Faktor 5-10 noch sehr geringe Ströme wie z. B. die der $\mathrm{C}_{2} \mathrm{H}_{5}{ }^{++}$-Ionen in Propan und n-Butan registriert und zur Gewinnung der AE-Verteilung differenziert werden konnten. Das Auflösungsvermögen der Anordnung war wegen der zur Vermeidung von $\mathrm{x}$-Ausblendung ${ }^{2}$ verbreiterten Spalte und infolge der hohen Ziehspannung relativ gering $(A=50-70)$, jedoch noch groß genug, daß bei den Ionen der $\mathrm{C}_{5}^{++}$-Gruppe eine gegenseitige Störung der AE-Verteilungen durch Überlagerung vernachlässigt werden konnte.

Die Messungen wurden hauptsächlich an der Reihe der n-Paraffine vom Propan bzw. Äthan bis zum n-Decan ausgeführt, dazu an Isomeren des Butans, Pentans und Hexans. Mehr der Übersicht dienten Messungen an den Butenen, dem 1,3-Butadien und dem 3-Methyl-Penten als Vertretern von nicht gesättigten Kohlenwasserstoffen. Die Reinheit dieser Substanzen war mit mindestens $99,8 \%$ (beim n-Decan mindestens $99,5 \%$ ) groß genug, um durch Verunreinigung hervorgerufene Verfälschungen der AE-Verteilungen und daraus resultierende Fehlschlüsse mit großer Wahrscheinlichkeit auszuschließen.

\begin{tabular}{|c|c|c|c|c|c|c|c|c|c|c|c|c|c|}
\hline \multicolumn{14}{|c|}{ der Satellitgruppen $(\mathrm{eV})$} \\
\hline \multicolumn{2}{|c|}{$\begin{array}{l}\text { 2-Methyl- } \\
\text { Pentan }\end{array}$} & \multicolumn{2}{|c|}{$\begin{array}{l}\text { 2,3-Dimethyl- } \\
\text { Butan }\end{array}$} & \multicolumn{2}{|c|}{ n-Heptan } & \multicolumn{2}{|c|}{ n-Octan } & \multicolumn{2}{|c|}{ n-Nonan } & \multicolumn{2}{|c|}{ n-Decan } & \multirow{2}{*}{ Ion } & \multirow[t]{2}{*}{$M$} \\
\hline 1.S & $2 . \mathrm{S}$ & 1.S & 2.S & $1 . \mathrm{S}$ & $2 . \mathrm{S}$ & $1 . \mathrm{S}$ & $2 . \mathrm{S}$ & 1.S & 2.S & 1.S & $2 . \mathrm{S}$ & & \\
\hline $\begin{array}{l}\text { a } \mathbf{2 , 5 8} \\
\text { a } \mathbf{2 , 3 0}\end{array}$ & $\begin{array}{l}\text { c } 5,49 \\
\text { b } 5,26\end{array}$ & $\begin{array}{l}\text { a } \mathbf{2 , 7 1} \\
\text { a } \mathbf{2 , 5 9}\end{array}$ & $\begin{array}{l}\text { c } 5,71 \\
\text { b } 5,48\end{array}$ & $\begin{array}{l}\text { a } \mathbf{2 , 3 5} \\
\text { a } \mathbf{2 , 1 4}\end{array}$ & $\begin{array}{l}\text { c 4,92 } \\
\text { b 4,68 }\end{array}$ & $\begin{array}{l}\text { b } \mathbf{2 , 3 7} \\
\text { a } \mathbf{2 , 1 0}\end{array}$ & $\begin{array}{l}\text { b 4,57 } \\
\text { b 4,46 }\end{array}$ & $\begin{array}{l}\text { b } \mathbf{2 , 4 9} \\
\text { b } \mathbf{2 , 0 9}\end{array}$ & $\begin{array}{l}\text { c 4,38 } \\
\text { b 4,13 }\end{array}$ & $\begin{array}{l}\text { b 2,53 } \\
\text { b 2,10 }\end{array}$ & $\begin{array}{c}(\mathrm{c} 4,52) \\
\mathrm{cs,92}\end{array}$ & $\begin{array}{l}\mathrm{CH}_{2}^{+} \\
\mathrm{CH}_{3}{ }^{+}\end{array}$ & $\begin{array}{l}14 \\
15\end{array}$ \\
\hline $\begin{array}{l}\text { b 2,20 } \\
\text { b 2,16 } \\
\text { b 2,21 } \\
\text { b 1,83 } \\
\text { b 2,19 }\end{array}$ & $\begin{array}{l}\text { (c } \overline{4,45}) \\
\text { c } 4,40 \\
\text { c } 4,42\end{array}$ & \begin{tabular}{|l|} 
b 2,18 \\
b 2,03 \\
\\
b 1,28s \\
b 2,24
\end{tabular} & $\begin{array}{c}(\mathrm{c} 4,15) \\
\ldots\end{array}$ & \begin{tabular}{|l|} 
b 1,88 \\
b 2,00 \\
b 1,87 \\
b 2,08 \\
c 0,71 s \\
a 1,84
\end{tabular} & \begin{tabular}{|l} 
c 4,02 \\
c 4,11 \\
c 4,32 \\
c 4,23 \\
c 4,33
\end{tabular} & & & $\begin{array}{l}\text { b } 1,75 \\
\text { b } 1,61 \\
\text { b } 1,67 \\
\text { a } 1,56\end{array}$ & $\begin{array}{l}\text { c } 3,85 \\
\text { c } 3,85 \\
\text { c } 3,48 \\
\text { c } 3,82\end{array}$ & & & $\begin{array}{l}\mathrm{C}_{2} \mathrm{H}^{+} \\
\mathrm{C}_{2} \mathrm{H}_{2} 2^{+} \\
\mathrm{C}_{2} \mathrm{H}_{3}{ }^{+} \\
\mathrm{C}_{2} \mathrm{H}_{4}{ }^{+} \\
\\
\mathrm{C}_{2} \mathrm{H}_{5}\end{array}$ & $\begin{array}{l}25 \\
26 \\
27 \\
28 \\
\\
29\end{array}$ \\
\hline $\begin{array}{l}\text { b } 1,84 \\
\text { b 1,82 } \\
\text { b 1,79 } \\
\text { b } 1,67 \\
\text { b } 1,74 \\
\text { b } 1,03 \mathrm{~s} \\
\text { a } 1,80\end{array}$ & $\begin{array}{l}- \\
- \\
- \\
- \\
- \\
-\end{array}$ & $\begin{array}{l}\text { b 2,07 } \\
\text { b } 1,89 \\
\text { a } 1,95 \\
\text { a 1,88 } \\
\text { a } 1,93\end{array}$ & $\begin{array}{l}- \\
- \\
- \\
- \\
-\end{array}$ & $\begin{array}{l}\text { b } 1,75 \\
\text { b } 1,75 \\
\text { c } 1,74 \\
\text { c } 1,65 \\
\text { c } 0,66 \mathrm{~s} \\
\text { c } 1,63 \\
\text { c } 1,84 \\
\text { c } 0,50 \mathrm{~s} \\
\text { b } 1,64\end{array}$ & $\begin{array}{c}- \\
- \\
- \\
\overline{(c} 4,36) \\
-\end{array}$ & & & & & & & $\begin{array}{l}\mathrm{C}_{3} \mathrm{H}^{+} \\
\mathrm{C}_{3} \mathrm{H}_{2}+ \\
\mathrm{C}_{3} \mathrm{H}_{3}^{+} \\
\mathrm{C}_{3} \mathrm{H}_{4}^{+} \\
\\
\mathrm{C}_{3} \mathrm{H}_{5}{ }^{+} \\
\mathrm{C}_{3} \mathrm{H}_{6} \\
\\
\mathrm{C}_{3} \mathrm{H}_{7}+\end{array}$ & $\begin{array}{l}37 \\
38 \\
39 \\
40 \\
\\
41 \\
42 \\
\\
\\
43\end{array}$ \\
\hline 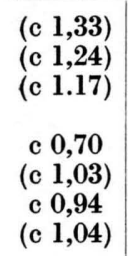 & & $\begin{array}{c}\mathrm{c} 0,60(\mathrm{~s})^{\mathrm{a}} \\
\text { b } 0, \dot{59}(\mathrm{~s}) \\
\text { c } 0,54(\mathrm{~s})\end{array}$ & & $\begin{array}{ll}\text { b } 1,38 \\
\text { c } 1,39 \\
\text { c } 1.34 \\
\text { c } 1.19 \\
\text { c } 1.01 \\
\text { c } 1,06 \\
\text { c } 1,03 \\
\text { b } 1,38\end{array}$ & $\begin{array}{l}- \\
- \\
- \\
- \\
-\end{array}$ & & & & & & & $\begin{array}{l}\mathrm{C}_{4} \mathrm{H}_{2}+ \\
\mathrm{C}_{4} \mathrm{H}_{3}^{+} \\
\mathrm{C}_{4} \mathrm{H}_{4^{+}} \\
\mathrm{C}_{4} \mathrm{H}_{5}^{+} \\
\mathrm{C}_{4} \mathrm{H}_{6}^{+} \\
\mathrm{C}_{4} \mathrm{H}_{7}^{+} \\
\mathrm{C}_{4} \mathrm{H}_{8}^{+} \\
\mathrm{C}_{4} \mathrm{H}_{9^{+}}^{+}\end{array}$ & $\begin{array}{l}50 \\
51 \\
52 \\
53 \\
54 \\
55 \\
56 \\
57\end{array}$ \\
\hline
\end{tabular}

der Verteilungsmaxima. Durch Druck hervorgehobene Satelliten sind relativ intensiv und machen mehr als $10 \%$ der Gesamtmit „... gekennzeichneten Ionen zeigen schwache, die mit „- “ gekennzeichneten innerhalb der Meßgenauigkeit keine AnBuchstaben $\mathbf{a}, \mathrm{b}$ und $\mathrm{c}$ charakterisieren wie in II die gegenseitige Trennung der Satellitgruppen; bei a und b ist das Höhe dieses Maximums. Im Falle c ist die Satellitgruppe nicht mehr durch ein Maximum hervorgehoben. s bedeutet: sekundärer Satelliten dieser Gruppe ist unsicher (siehe Text).

9 Fernseh-AG, Darmstadt. 


\section{Ergebnisse}

In den Tab. 1 und 2 sind die an den Stellen der Verteilungsmaxima gemessenen Anfangsenergien ${ }^{\text {w }} E$ der beobachteten 1. und 2. Satellitgruppen bei ein-

\begin{tabular}{|c|c|c|c|}
\hline \multirow[t]{2}{*}{ Substanz } & \multirow[t]{2}{*}{ Ion } & \multicolumn{2}{|c|}{$\begin{array}{c}\text { Satellit- } \\
\text { energien }(\mathrm{eV})\end{array}$} \\
\hline & & 1.S & $2 . \mathrm{S}$ \\
\hline $\begin{array}{l}\text { Buten-1 } \\
\text { cis-Buten-2 } \\
\text { trans-Buten-2 } \\
\text { iso-Buten } \\
\text { 3-Methyl-Buten-1 } \\
\text { Butadien-1,3 } \\
\text { Butadien-1,3 }\end{array}$ & $\begin{array}{l}\mathrm{CH}_{3}{ }^{+} \\
\mathrm{CH}_{3}{ }^{+} \\
\mathrm{CH}_{3}{ }^{+} \\
\mathrm{CH}_{3}{ }^{+} \\
\mathrm{CH}_{3}{ }^{+} \\
\mathrm{CH}_{3}{ }^{+} \\
\mathrm{CH}_{2}{ }^{+}\end{array}$ & $\begin{array}{l}\text { a } \mathbf{2 , 6 1} \\
\text { a } \mathbf{2 , 5 8} \\
\text { a } \mathbf{2 , 5 5} \\
\text { a } \mathbf{2 , 7 4} \\
\text { a } \mathbf{2 , 5 9} \\
\text { a } \mathbf{2 , 5 4} \\
\text { a } \mathbf{2 , 7 3}\end{array}$ & $\begin{array}{lc}\text { b } & 5,75 \\
\text { b } & 5,58 \\
\text { b } & 5,47 \\
\text { b } & 5,96 \\
\text { b } & 5,87 \\
\text { c } & (5,58) \\
\text { c } & 5,89\end{array}$ \\
\hline
\end{tabular}

Tab. 2. Anfangsenergien der 1. und 2. Satellitgruppen bei den $\mathrm{CH}_{3}{ }^{+}$- bzw. $\mathrm{CH}_{2}{ }^{+}$-Ionen von ungesättigten Kohlenwasserstoffen. fachgeladenen und in Tab. 3 die Anfangsenergien der Satelliten bei doppeltgeladenen Ionen zusammengestellt. Die Anfangsenergien der 1. Satelliten solcher Ionen, bei denen keine 2 . Satelliten gefunden wurden, sind zur Ergänzung und zur Kontrolle der früheren Messungen (II) mit aufgeführt, wobei die alten Werte für einen Vergleich um rund $8 \%$ anzuheben sind (siehe Abschn. 1). Aus Tab. 1 ist zu ersehen, daß es sich bei dem Auftreten der schon in IV besprochenen sekundären Satelliten bei den $\mathrm{C}_{2} \mathrm{H}_{4}{ }^{+}$und $\mathrm{C}_{3} \mathrm{H}_{6}{ }^{+}$-Ionen, die sich offenbar durch eine besonders stabile Konfiguration hervorheben, um eine mehr regelmäßige als zufällige Erscheinung handelt. Unter Umständen kann das Fehlen von sekundären Satelliten auch strukturbedingt sein: So wäre es z. B. für das Auftreten solcher Satelliten unter den $\mathrm{C}_{3} \mathrm{H}_{6}{ }^{+}$. Ionen des 2,3-Dimethylbutans nötig, daß entweder

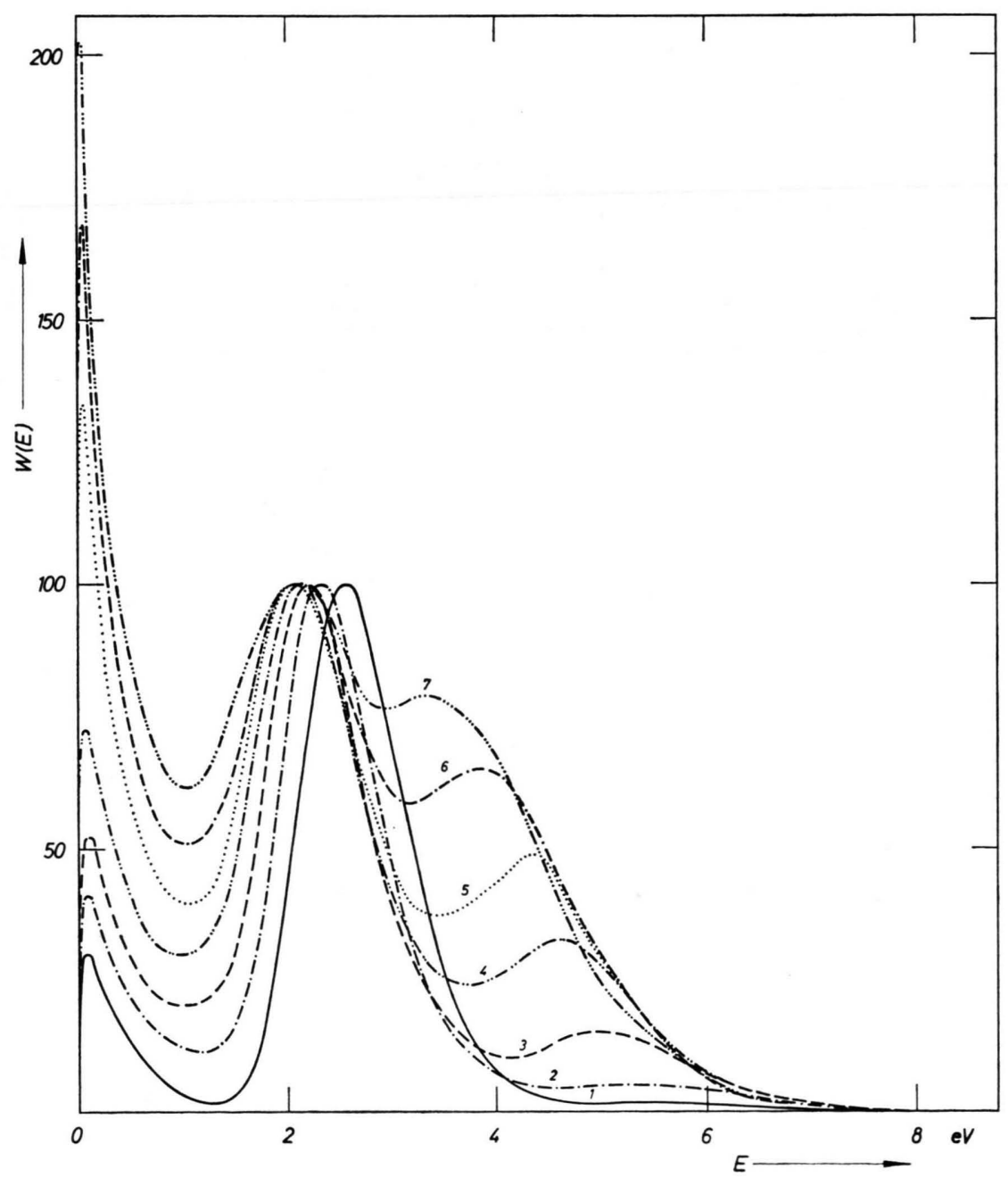

Abb. 1.

AE-Verteilungen $W(E)$ der $\mathrm{CH}_{3}{ }^{+}$-Ionen bei den $n$-Paraffinen, aufgetragen über der Anfangsenergie $E$. Die Höhen der Maxima der 1. Satelliten sind willkürlich gleich 100 gesetzt. Die Ziffern an den Kurven kennzeichnen die einzelnen Paraffine:

1) n-Butan,

2) n-Pentan,

3) n-Hexan,

4) n-Heptan,

5) n-Octan,

6) n-Nonan und

7) n-Decan. 
schon im Molekülion eine Bindungsumordnung eintritt oder aber drei statt zwei C - C-Bindungsbrüche erfolgen, von denen der erste mit einer Ladungstrennung verbunden ist. Solche Prozesse dürften aber gerade beim Zerfall zweifachgeladener Molekülionen nach den bisherigen Erfahrungen zumindest recht selten sein. Ähnliche Argumente führen zu einer Erklärung für die beim 2,3-Dimethyl-Butan gegenüber den anderen beiden Hexanen wesentlich geringeren Energie der Satelliten in der $\mathrm{C}_{4}{ }^{+}$-Gruppe: Auch hier kommt infolge der verzweigten Struktur ein einfacher ladungstrennender $\mathrm{C}-\mathrm{C}$-Bindungsbruch allein als Entstehungsursache nicht in Frage. Es wird sich bei diesen z. Tl. relativ intensiven Satelliten - bei den $\mathrm{C}_{4} \mathrm{H}_{6}{ }^{+}$-Ionen macht der Satellit mehr als das doppelte der quasithermischen Gruppe aus - also entweder um „echte" sekundäre Satelliten (erst ladungstrennender $\mathrm{C}-\mathrm{C}$-Bindungsbruch, dann neutrale CGruppenabspaltung) handeln oder aber um Gruppen, die dadurch gebildet werden, daß zunächst eine neutrale $\mathrm{C}_{1}$-Gruppe abgespalten wird und dann der ladungstrennende Proze $\beta$ von einer $\mathrm{C}_{5}{ }^{++}$-Gruppe (Pentanstruktur) aus stattfindet. Beide Möglichkeiten liefern Satellitenergien gleicher Größenordnung, so daß eine eindeutige Entscheidung nicht möglich ist.

Aus Tab. 1 geht nun weiter hervor, daß die 2. Satelliten bei den Paraffinen in der $\mathrm{C}_{1}{ }^{+}$-Gruppe nicht nur vereinzelt auftreten, sondern, wenigstens vom
Butan an aufwärts, eine allgemeine Erscheinung darstellen, wobei am willkürlich herausgegriffenen Beispiel des 3-Methyl-Pentans beobachtet wurde, daß auch unter den $\mathrm{C}^{+}$- und $\mathrm{CH}^{+}$-Ionen (in der Tabelle nicht angegeben) solche relativ energiereichen Gruppen vorhanden sind. Abb. 1 zeigt anschaulich, wie bei den $\mathrm{CH}_{3}{ }^{+}$-Ionen der n-Paraffine die Intensität der 2. Satelliten mit zunehmender Größe des Moleküls anwächst.

Bei den Paraffinen werden vom Pentan an aufwärts auch in der $\mathrm{C}_{2}{ }^{+}$-Gruppe solche zweiten Satelliten neben den ersten mit zunehmender Regelmäßigkeit gefunden. $\mathrm{Da}$ es sich dabei wirklich um 2 . und 1. Satelliten handelt, geht sowohl aus den Energien als auch aus den Verteilungen $\operatorname{der} \mathrm{C}_{2} \mathrm{H}_{4}{ }^{+}$-Ionen bei einigen Paraffinen hervor, wo gleichzeitig 2., 1. und sekundäre Satelliten vorhanden sind. In $\operatorname{der} \mathrm{C}_{3}{ }^{+}$Gruppe dagegen zeigten sich nur bei den $\mathrm{C}_{3} \mathrm{H}_{6}{ }^{+}$Ionen des n-Hexans und n-Heptans Andeutungen einer weiteren energiereichen Gruppe (Abb. 2). Von den Satelliten bei doppeltgeladenen Ionen (Tab. 3) waren überall besonders die der $\mathrm{C}_{3}{ }^{++}$-Gruppe stark ausgeprägt und gut zu messen, wenngleich die $\mathrm{Ab}$ solutintensität dieser Ionen im Spektrum sehr gering ist. Abb. 3 zeigt als Beispiel die Verteilungen einiger Ionen dieser Gruppe aus verschiedenen Substanzen.

In der $\mathrm{C}_{4}{ }^{++}$-Gruppe konnte vom n-Pentan an aufwärts nur bei den $\mathrm{C}_{4} \mathrm{H}_{3}{ }^{++}$-Ionen (Massenzahl 25,5), die auch in den API-Spektren ${ }^{10}$ als einzige Vertre-

\begin{tabular}{|c|c|c|c|c|c|c|c|c|c|c|c|c|c|}
\hline \multirow{3}{*}{$M / q$} & \multirow{3}{*}{ Ion } & \multicolumn{11}{|c|}{ Anfangsenergien der Satellitgruppen $(\mathrm{eV})$} & \multirow{3}{*}{$M / q$} \\
\hline & & & $\exists$ & & శ్జ & $\approx$ & 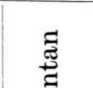 & & $\frac{1}{D}$ & $\frac{\dot{d}}{\vec{D}}$ & \multicolumn{2}{|c|}{ n-Heptan } & \\
\hline & & مْ & 竞 & 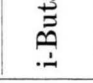 & 巳̈ & . & 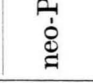 & $\stackrel{0}{\stackrel{0}{I}}$ & 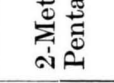 & 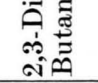 & 1. $\mathrm{S}$ & 2. $\mathrm{S}$ & \\
\hline 14,5 & $\mathrm{C}_{2} \mathrm{H}_{5}++$ & a $\mathbf{2 , 6 9}$ & a $\mathbf{3 , 2 5}$ & & & & & & & & & & 14,5 \\
\hline $\begin{array}{l}19 \\
19,5 \\
20 \\
20,5\end{array}$ & $\begin{array}{l}\mathrm{C}_{3} \mathrm{H}_{2}{ }^{++} \\
\mathrm{C}_{3} \mathrm{H}_{3}{ }^{++} \\
\mathrm{C}_{3} \mathrm{H}_{4}{ }^{++} \\
\mathrm{C}_{3} \mathrm{H}_{5}{ }^{++}\end{array}$ & & $\begin{array}{l}\text { a } 1,87 \\
\text { a } 1,98 \\
\text { a } 2,01 \\
\text { a } 1,85\end{array}$ & $\begin{array}{ll}\text { a } & \mathbf{2 , 0 4} \\
\text { a } & \mathbf{2 , 0 0} \\
\text { a } 2,10 \\
\text { a } 2,03\end{array}$ & $\begin{array}{l}\text { a } \mathbf{2 , 5 9} \\
\text { a } \mathbf{2 , 6 0} \\
\text { a } \mathbf{2 , 6 5} \\
\text { a } \mathbf{2 , 5 8}\end{array}$ & $\begin{array}{l}\text { a } \mathbf{2 , 7 0} \\
\text { a } 2,80 \\
\text { a } \mathbf{2 , 8 2} \\
\text { a } \mathbf{2 , 7 4}\end{array}$ & 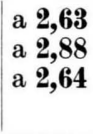 & $\begin{array}{l}\text { a } \mathbf{2 , 8 3} \\
\text { a } \mathbf{2 , 8 5} \\
\text { a } \mathbf{2 , 8 1} \\
\text { a } \mathbf{2 , 6 8}\end{array}$ & $\begin{array}{l}\text { a } 3,07 \\
\text { a } 3,05 \\
\text { b } 3,10 \\
\text { b } \mathbf{2 , 9 7}\end{array}$ & $\begin{array}{l}\text { a } \mathbf{3 , 3 2} \\
\text { a } \mathbf{3 , 2 8} \\
\text { a } \mathbf{3 , 3 2} \\
\text { a } \mathbf{3 , 3 1}\end{array}$ & $\begin{array}{|lr|}\text { a } & \mathbf{3 , 0 5} \\
\text { a } & \mathbf{2 , 8 6} \\
\text { a } & \mathbf{2 , 9 4} \\
\text { b } & \mathbf{2 , 7 1}\end{array}$ & $\begin{array}{c}\text { c } 6,17 \\
\text { (c } 5,43) \\
\text { b } 6,08 \\
\text { (c } 5,73)\end{array}$ & $\begin{array}{l}19 \\
19,5 \\
20 \\
20,5\end{array}$ \\
\hline 25,5 & $\mathrm{C}_{4} \mathrm{H}_{3}{ }^{++}$ & & & & b 1,48 & b 1,51 & b 1,48 & a 2,06 & b 2,04 & $\cdots$ & b 2,45 & & 25,5 \\
\hline $\begin{array}{l}31 \\
31,5 \\
32 \\
32,5 \\
33\end{array}$ & $\begin{array}{l}\mathrm{C}_{5} \mathrm{H}_{2}{ }^{++} \\
\mathrm{C}_{5} \mathrm{H}_{3}++ \\
\mathrm{C}_{5} \mathrm{H}_{4}^{++} \\
\mathrm{C}_{5} \mathrm{H}_{5}^{++} \\
\mathrm{C}_{5} \mathrm{H}_{6}{ }^{++}\end{array}$ & & & & & & & $\begin{array}{c}\text { c } 1,07 \\
(\mathrm{c} 1,06) \\
\text { (c } 0,93) \\
\text { (c } 1,22) \\
\text { (c } 1,09)\end{array}$ & $\begin{array}{c}\text { c } 1,07 \\
\text { c } 1,10 \\
\text { c } 1,14 \\
(\text { c } 1,10) \\
\ldots\end{array}$ & $\begin{array}{c}\text { c } 1,22 \\
\text { c } 1,10 \\
\text { c } 1,06 \\
\\
\ldots\end{array}$ & $\begin{array}{c}\text { b } 1,75 \\
\text { b } 1,81 \\
\text { c } 1,60 \\
\quad \ldots \\
\ldots\end{array}$ & & $\begin{array}{l}31 \\
31,5 \\
32 \\
32,5 \\
33\end{array}$ \\
\hline
\end{tabular}

Tab. 3. Anfangsenergien der Satellitgruppen bei doppeltgela denen Bruchstück-Ionen. $q=$ Zahl der mit einem Ion verbundenen Elementarladungen.

10 Mass Spectral Data, American Petroleum Institute (API) Research Project 44. 


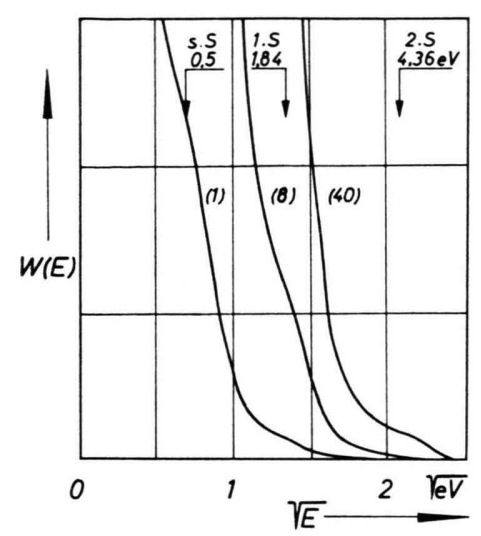

Abb. 2. AE-Verteilung $W(E)$ der $\mathrm{C}_{3} \mathrm{H}_{6}{ }^{+}$-Ionen in n-Heptan oberhalb $0,3 \mathrm{eV}$, aufgetragen über $V E$. Die Pfeile geben die Lage der einzelnen Satellitmaxima an, die mit s. S=sekundärer Satellit ${ }^{1}$, 1.S und 2.S gekennzeichnet sind. Die Zahlen an den verschiedenen Kurvenzügen geben die gegenseitige Überhöhung an.

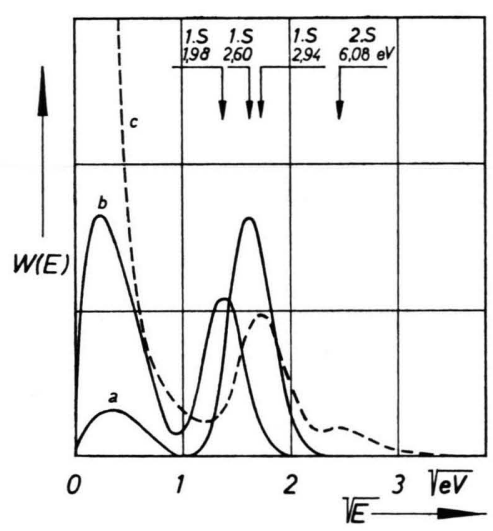

Abb. 3. Beispiele für AE-Verteilungen $W(E)$ in der $\mathrm{C}_{3}{ }^{++}$. Gruppe, aufgetragen über $\sqrt{E}$. Die Pfeile geben die Lage und die Energien der Satellitmaxima an. Es bedeuten: a) n-Pen$\tan \left(\mathrm{C}_{3} \mathrm{H}_{3}{ }^{++}\right)$, b) n-Butan $\left(\mathrm{C}_{3} \mathrm{H}_{3}{ }^{++}\right)$und c) n-Heptan $\left(\mathrm{C}_{3} \mathrm{H}_{4}{ }^{++}\right)$.

ter der doppeltgeladenen $\mathrm{C}_{4}$-Gruppe aufgeführt sind, eine Satellitgruppe gefunden werden. Zwar konnte wegen des geringen Auflösungsvermögens und der doch noch recht beachtlichen Druckverbreiterung der viel intensiveren benachbarten Linien $\operatorname{der} \mathrm{C}_{2} \mathrm{H}^{+}$- bzw. $\mathrm{C}_{2} \mathrm{H}_{2}{ }^{+}$-Ionen im Massenspektrum ein Maximum bei der Massenzahl 25,5 nicht festgestellt werden, doch hat die 1. Satellitgruppe der bei dieser Massenzahl aufgenommenen Verteilungskurve eindeutig eine andere Lage als die Satellitgruppen bei den Verteilungen der Massenzahlen 25 und 26, so daß es sich ohne Zweifel um doppeltgeladene Bruchstücke und nicht um gestreute $\mathrm{C}_{2} \mathrm{H}^{+}$- bzw. $\mathrm{C}_{2} \mathrm{H}_{2}{ }^{+}$-Ionen hoher Anfangsenergie handelt. Als Beispiel zeigt Abb. 4 die gemessenen Verteilungskurven bei den Massenzahlen $25\left(\mathrm{C}_{2} \mathrm{H}^{+}\right), 25,5\left(\mathrm{C}_{4} \mathrm{H}_{3}{ }^{++}\right)$und $26\left(\mathrm{C}_{2} \mathrm{H}_{2}{ }^{+}\right)$ in n-Hexan. Der angedeutete „2. Satellit“ in der Verteilung der Massenzahl 25,5 rührt in Wirklichkeit von der erwähnten Streuung her. Die quasithermische Gruppe interessiert hier nicht.

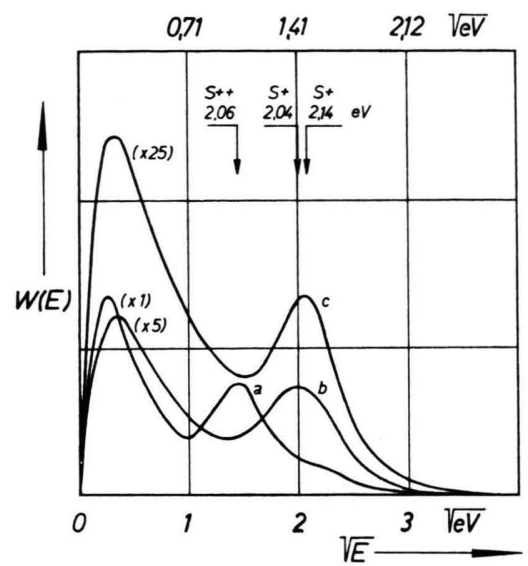

Abb.4. AE-Verteilungen $W(E)$ bei den Massenzahlen 25,5 (a), 25 (b) und 26 (c) im Massenspektrum des n-Hexans, aufgetragen über $\sqrt{E}$. Die $V E$-Skala für die einfachgeladenen Ionen $\mathrm{C}_{2} \mathrm{H}^{+}$und $\mathrm{C}_{2} \mathrm{H}_{2}{ }^{+}$befindet sich am oberen, die für die doppeltgeladenen Ionen $\mathrm{C}_{4} \mathrm{H}_{3}{ }^{++}$am unteren Rand der Abbildung. Die Pfeile geben die Lage und die Energien der Satellitmaxima an. Die Zahlen in Klammern geben die Faktoren an, mit denen die Ordinaten der einzelnen Kurven für einen Intensitätsvergleich multipliziert werden müssen.

Bei den Ionen der $\mathrm{C}_{5}{ }^{++}$-Gruppe findet man erst beim Heptan Satellitgruppen mit einem Maximum in der Verteilungskurve, während sie bei den Hexanen weniger ausgeprägt erscheinen (Abb. 5). In der $\mathrm{C}_{6}$-Gruppe wurden beim n-Heptan keine doppeltgeladenen Ionen gefunden.

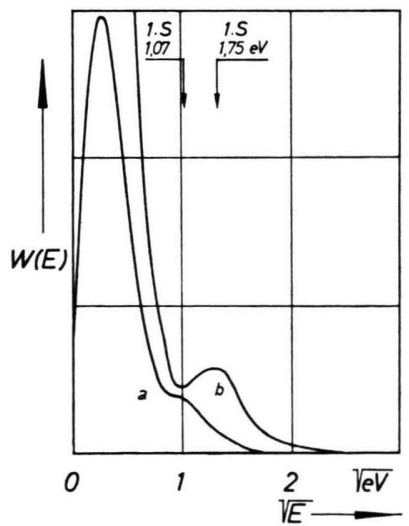

Abb. 5. Beispiele für AE-Verteilungen $W(E)$ in der $\mathrm{C}_{5}^{++}$. Gruppe, aufgetragen über $\sqrt{E}$. Die Pfeile geben die Lage und die Energien der Satellitmaxima an. Es bedeuten: a) 2-Methylpentan $\left(\mathrm{C}_{5} \mathrm{H}_{2}{ }^{++}\right)$und b) n-Heptan $\left(\mathrm{C}_{5} \mathrm{H}_{2}^{++}\right)$. 
Nach den Tab. $1-3$ fällt nun auf, daß einmal bei den einfachgeladenen Ionen die Energien der 2. Satelliten im allgemeinen nur wenig mehr als das Zweifache der Energien der 1. Satelliten betragen und $\mathrm{da}$ zum anderen auch bei den doppeltgeladenen Ionen die Satellitenergien fast doppelt so groß sind wie die der einfachgeladenen Bruchstücke mit gleicher Kohlenstoffzahl. Außerdem sind sie - wie bei den in IV besprochenen 1. Satelliten - für Ionen derselben $\mathrm{C}^{+}$- bzw. $\mathrm{C}^{++}$-Gruppe auch annähernd gleich.

Dies führt zu der Vermutung, daß diesen „neuen“ Satellitgruppen ein ähnlicher Entstehungsmechanismus zugrunde liegt, wie er schon bei den bisher gefundenen 1. Satelliten postuliert worden war, nämlich, daß es sich hier um den Zerfall eines dreifachgeladenen Molekülions ${ }^{11}$ in ein einfach- und ein zweifachgeladenes Bruchstück handelt.

Eine wichtige Vorausestzung für die Gültigkeit dieser Hypothese ist wieder die Erfüllung des Impulssatzes, hier nun zwischen Bruchstücken mit verschiedener Ladung. Da jedoch die Zerfallsprozesse und -wege im einzelnen nicht bekannt sind und die Möglichkeit besteht, daß durch Dissoziation eines Molekülions entstandene und zusammengehörige Bruchstückionen vor ihrem Nachweis noch H-Atome bzw. $\mathrm{H}_{2}$-Moleküle abspalten, wird man keine exakte Erfüllung des Impulssatzes erwarten können, sondern man muß sich mit einer angenäherten Impulsgleichheit zwischen den entsprechenden Satelliten korrelierter C-Gruppen begnügen. In Tab. 4 sind die den Impulsen proportionalen Werte $\sqrt{ }^{\overline{\mathrm{w}} E M^{12}}$ für die 2. Satellitgruppen bei einfachgeladenen und für die Satellitgruppen bei doppeltgeladenen Bruchstücken zusammengestellt.

Die Werte in eckigen Klammern sind aus den gemessenen Impulsen für den möglichen Fall berechnet, daß der ladungstrennende Proze $ß$ zunächst durch einen einfachen C-C-Bindungsbruch am dreifachgeladenen Molekülion erfolgt und die so gebildeten einfach- und doppeltgeladenen Bruchstücke der Form $\mathrm{C}_{i} \mathrm{H}_{2 i+1}{ }^{+}$und $\mathrm{C}_{n-i} \mathrm{H}_{2(n-i)+1}{ }^{++}$dann durch $\mathrm{H}_{2}$ - bzw. $\mathrm{H}$-Abspaltung in die nachgewiesenen Ionen übergehen. Mit der Annahme, daß die bei dieser neu-

11 Über die Existenz von stabilen dreifach geladenen Ionen bei organischen Substanzen siehe: a) S. Meyerson u. R. W. van der HaAr, J. Chem. Phys. 37, 2458 [1962] und b) F. H. Dorman u. J. D. Morrison, J. Chem. Phys. 35, 575 [1961].

12 Siehe auch IV, Tab. 1. tralen Wasserstoffabspaltung ${ }^{13}$ freiwerdende Ubergangsenergie zu vernachlässigen ist, würden die eingeklammerten Werte bei dieser Aufeinanderfolge der Prozesse dann die Impulse für die primär gebildeten Bruchstück-Ionen darstellen. Erfolgt die gesamte Wasserstoffspaltung vor dem ladungstrennenden Prozeß, so sind natürlich die gemessenen Werte für den ladungstrennenden Prozeß maßgebend. In jedem anderen Fall liegen, wenn man von einer Dissoziation in drei einfachgeladene Teilchen einmal absieht, die Impulse der unmittelbar bei der Ladungstrennung entstehenden Ionen, auf die es bei einem exakten Impulsvergleich ankäme, zwischen diesen beiden Grenzwerten.

Im Hinblick auf diese Entstehungshypothese sollen die Ergebnisse bei den einzelnen Paraffinen kurz besprochen werden:

Äthan und Propan. Beim Äthan konnte in der $\mathrm{C}_{1}$ Gruppe auch bei Elektronenenergien bis etwa $300 \mathrm{eV}$ keinerlei 2. Satellit entdeckt werden. Beim Propan ist eine solche Ionengruppe bei der Massenzahl 15 wenigstens, wenn auch nur schwach, angedeutet und geht in den Ausläufer des 1. Satelliten über. Daneben wurde im Propanspektrum jedoch auch bei der Massenzahl 14,5 eine Linie gefunden, deren Intensität zwar äußerst gering war, die sich aber hauptsächlich aus $\mathrm{C}_{2} \mathrm{H}_{5}{ }^{++}$-Ionen hoher Anfangsenergie zusammensetzte. Aus Abb. 6, wo die Intensitätsverteilung der Linie bei Massenzahl 14,5 über der zu $\sqrt{E}$ proportionalen Spannung am Ablenkkondensator $U_{\mathrm{d}}$ (siehe auch II) aufgezeichnet ist, ist dies unmittelbar zu ersehen. Die beiden Satelliten haben

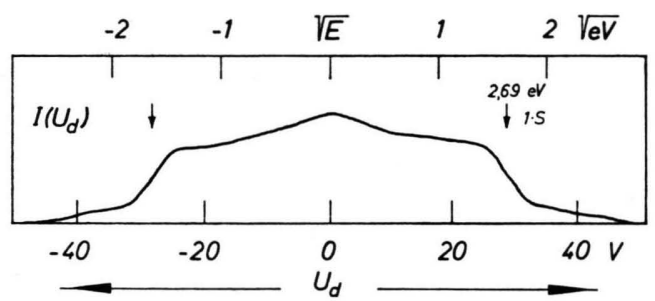

Abb. 6. Intensitätsverteilung der $\mathrm{C}_{2} \mathrm{H}_{5}{ }^{++}$-Ionen $(M / q$ 14,5) im Propanspektrum in Abhängigkeit von der zu $V E$ proportionalen Spannung $U_{\mathrm{d}}$ am Ablenkkondensator. Die Ausläufer der Kurve für $\left|U_{\mathrm{d}}\right| \geqq 30 \mathrm{~V}$ sind durch gestreute $\mathrm{CH}_{3}{ }^{+}$- und $\mathrm{CH}_{2}{ }^{+}$-Ionen hoher Anfangsenergie verursacht.

13 Eine Verknüpfung der hier besprochenen Satelliten mit ladungstrennenden Wasserstoffspaltungen - ob vor oder ob nach Abspaltung einer neutralen C-Gruppe - ist wegen der zu hohen Energien, die die $\mathrm{H}_{2}{ }^{+}$- bzw. $\mathrm{H}^{+}$-Ionen dann haben müßten, undenkbar. 


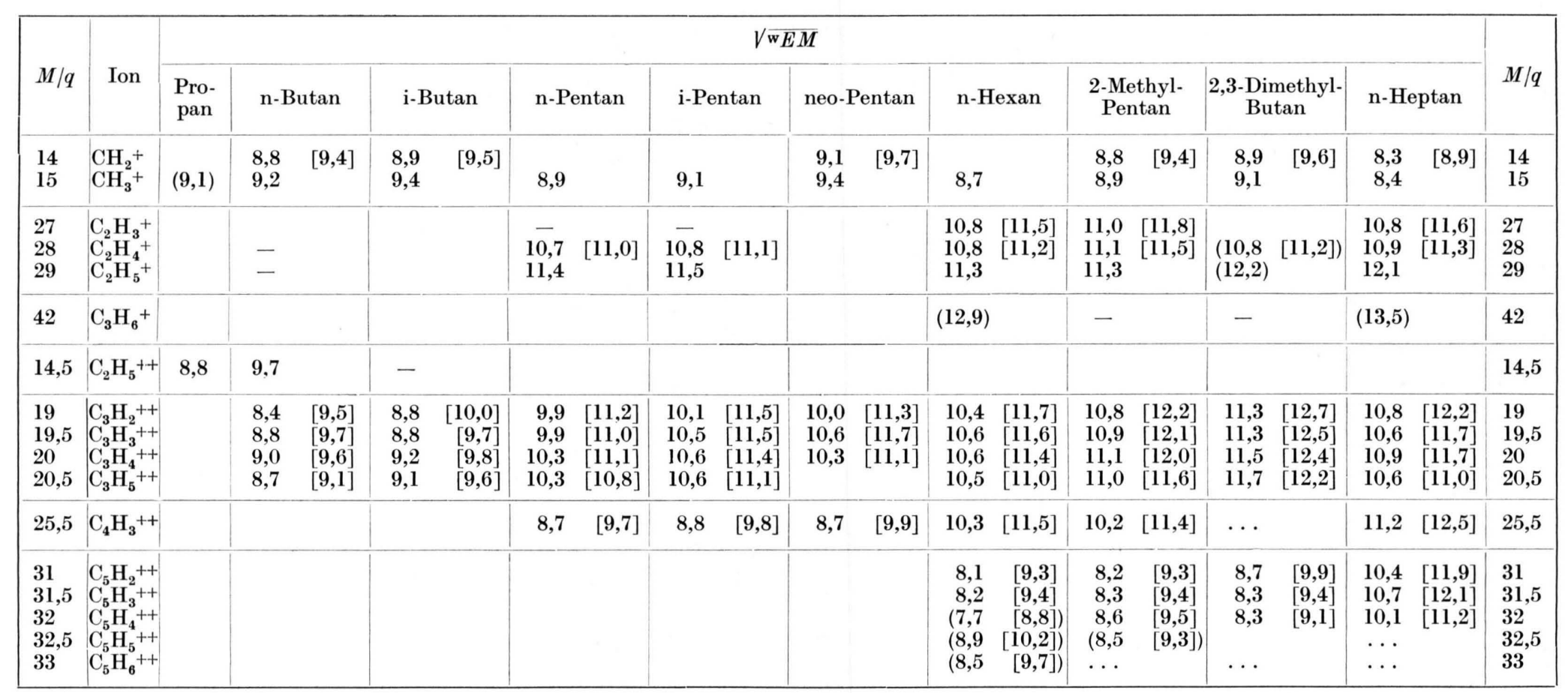

Tab. 4. Impulse $V^{\sqrt{w} E M}$ für 2. Satelliten bei einfachgeladenen und für 1 . Satelliten bei doppeltgeladenen Ionen; ${ }^{\text {w }} E=$ Anfangsenergie, gemessen an der Stelle des Maximums der Satellit-Verteilung, $M=$ Massenzahl, $q=$ Zahl der mit einem Ion verbundenen Elementarladungen. In den eckigen Klammern sind die Impulse angegeben, die die aus primären ladungstrennenden Prozessen stammenden Ionen $\mathrm{C}_{i} \mathrm{H}_{2 i+1}{ }^{+}$bzw. $\mathrm{C}_{j} \mathrm{H}_{2 j+1}{ }^{++}$unter der Voraussetzung haben würden, daß die nachgewiesenen Ionen $\mathrm{C}_{i} \mathrm{H}_{2 i+1-k^{+}}$bzw. $\mathrm{C}_{j} \mathrm{H}_{2 j+1-l}(k, l=1,2, \ldots)$ daraus durch nachträgliche $\mathrm{H}$ - bzw. $\mathrm{H}_{2}$-Abspaltung entstehen (s. Text S. 2075). 
nach Tab. 4 nun annähernd gleichen Impuls, sind also mit großer Wahrscheinlichkeit zueinander korreliert. Da es sich bei der Entstehung der beiden Bruchstücke $\mathrm{C}_{2} \mathrm{H}_{5}^{++}$und $\mathrm{CH}_{3}{ }^{+}$nach unserer Hypothese um die primäre Dissoziation eines Molekülions handelt, sollten die Impulse exakt gleich sein. Gerade hier ist aber die zur Festlegung des Maximums des 2. Satelliten bei den $\mathrm{CH}_{3}{ }^{+}$-Ionen nötige Extrapolation relativ unsicher.

Butane. Hier sind die 2. Satelliten in $\operatorname{der} \mathrm{C}_{1}{ }^{+}$. Gruppe, gemessen an den 1. Satelliten, zwar noch recht intensitätsarm, jedoch schon so deutlich davon getrennt, daß bei der Massenzahl 15 in der AE-Verteilung ein zweites Maximum auftritt. Bei den Linien der dazu korrelierten $\mathrm{C}_{3}{ }^{++}$-Gruppe $(M=19-20,5)$ sind die Ionen hoher Anfangsenergie sogar mindestens ebenso häufig wie die quasithermischen. Die Impulse beider Gruppen stimmen ebenfalls wieder in guter Näherung überein.

Beim n-Butan findet sich bei der Massenzahl 14,5 eine, ähnlich wie beim Propan, überwiegend aus $\mathrm{C}_{2} \mathrm{H}_{5}^{++}$. Ionen hoher Anfangsenergie bestehende Linie geringer Intensität, sie fehlt jedoch beim iso-Butan. Die Energie dieses Satelliten ist aber merklich niedriger als das Zweifache der Energien der 1 . Satelliten in der $\mathrm{C}_{2}^{+}$. Gruppe im Gegensatz zu den Energierelationen zwischen den 1. Satelliten in den $\mathrm{C}_{3}{ }^{+}$- und $\mathrm{C}_{3}{ }^{++}$-Gruppen der Butane. Bildet man jedoch für die $\mathrm{C}_{2}{ }^{+}$-Gruppen der n-Paraffine die mittleren Verhältnisse der Energien der 2. und 1. Satelliten, so nehmen diese vom n-Nonan bis zum n-Pentan herunter immer mehr ab und lassen sich, als Kurve über der Kohlenstoffzahl des Moleküls aufgetragen, zwanglos bis zu dem Wert extrapolieren, den man für das n-Butan unter der Annahme erhält, daß ein 2. Satellit in der $\mathrm{C}_{2}{ }^{+}$-Gruppe den gleichen Impuls und somit gleiche Energie wie der 1. Satellit bei den

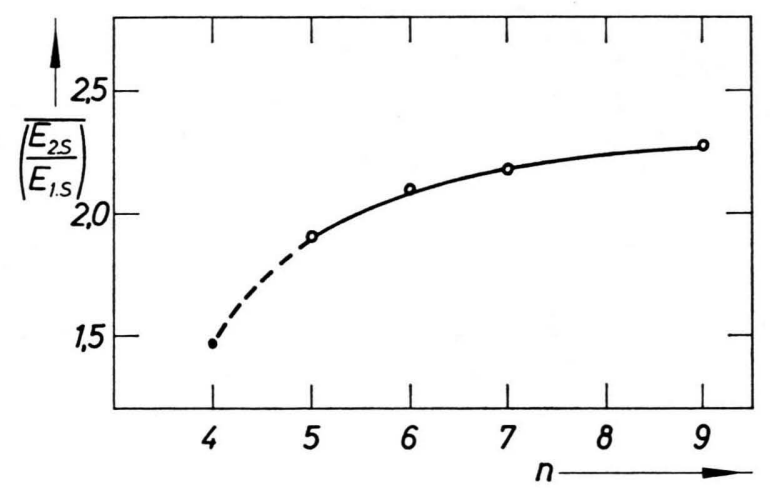

Abb. 7. Mittlere Verhältnisse der Energien der 2. und 1. Satelliten in den $\mathrm{C}_{2}{ }^{+}$-Gruppen bei verschiedenen n-Paraffinen (gekennzeichnet durch die Kohlenstoffzahl $n$ ). Der Punkt für $\mathrm{n}$-Butan ist unter der im Text erläuterten Annahme berechnet.
$\mathrm{C}_{2} \mathrm{H}_{5}^{++}$-Ionen haben würde (Abb. 7). Auch die Tatsache, daß in der $\mathrm{C}_{2}^{+}$-Gruppe von $\mathrm{n}$-Butan keine 2 . Satelliten gefunden werden konnten, wäre damit erklärt, denn solche, intensitätsmäßig recht schwache Gruppen, werden hier durch die Ausläufer der viel intensiveren 1. Satelliten überdeckt. Es spricht also nach den vorliegenden Ergebnissen trotz des anfänglichen Anscheins nichts dagegen, daß auch dieser etwas vereinzelt dastehende $\mathrm{C}_{2} \mathrm{H}_{5}^{++}$-Satellit durch den Zerfall eines dreifachgeladenen Molekülions entsteht. Das Fehlen dieser energiereichen $\mathrm{C}_{2} \mathrm{H}_{5}^{++}$-Ionen beim iso-Butan hat seine Parallele in der - gegenüber dem n-Butan - viel geringeren Intensität bei den 1 . Satelliten $\operatorname{der} \mathrm{C}_{2}^{+}$-Gruppe (siehe IV), da solche Ionengruppen - natürlich immer unter der Voraussetzung der obigen Hypothese - in beiden Fällen nur über eine Bindungsumordnung entstehen können.

Die Aufstellung einer Kohlenstoffbilanz wurde nur beim n-Butan versucht, da in diesem Fall die Verhältnisse noch recht einfach erschienen. Doch die Gesamtintensität der Satellitionen in der $\mathrm{C}_{3}{ }^{++}$-Gruppe beträgt hier weniger als $50 \%$ der Intensität der 2 . Satellitgruppe bei den $\mathrm{CH}_{3}{ }^{+}$-Ionen. Wie in IV ergibt sich also eine Unterbilanz auf der Seite höherer Massen und man ist geneigt, dies durch einen Abbau der Ionen der $\mathrm{C}_{3}{ }^{++}$-Gruppe z. B. durch eine weitere Ladungstrennung zu erklären oder durch solche Zerfälle des dreifachgeladenen Molekülions, bei denen gleichzeitig drei einfachgeladene Teilchen entstehen, wobei gerade beim Butan bevorzugt energiereiche $\mathrm{C}_{1}{ }^{+}$-Ionen gebildet werden. Über das Vorkommen solcher Prozesse läßt sich jedoch keine definierte Aussage machen: Bei einem Weiterzerfall von $\mathrm{C}_{3}{ }^{++}$. Ionen in $\mathrm{C}_{2}{ }^{+}$-Ionen und $\mathrm{C}_{1}{ }^{+}$-Ionen würde sich $\mathrm{z}$. $\mathrm{B}$. für jede dieser letzteren Ionengruppen eine sehr breit verschmierte Energieverteilung ergeben, die in der $\mathrm{C}_{2}{ }^{+}$-Gruppe unter den Ausläufern der 1. Satelliten, in der $\mathrm{C}_{1}{ }^{+}$-Gruppe unter den 2. Satelliten verschwinden würde, während bei einem Dreierzerfall des Molekülions sich überhaupt nichts über die Energieaufteilung vorhersagen läßt. Schließlich besteht, wie Ehrhardt und TekaAt ${ }^{14}$ bei den 1. Satelliten durch Auftrittspotentialmessungen am Beispiel des Propans gezeigt haben, noch die Möglichkeit, $\mathrm{da} ß$ Ionen hoher Anfangsenergie auch aus nichtladungstrennenden Prozessen stammen können. Ebenso wie in IV können also aus der Kohlenstoffbilanz keine eindeutigen Schlüsse gezogen werden. Das für n-Butan Gesagte gilt sinngemäß für alle untersuchten Substanzen.

14 H. Ehrhardt u. T. TekaAt, Z. Naturforschg. 19a, 1382 [1964]. 
Pentane. Bei den Pentanen sind zu den 2. Satelliten in der $\mathrm{C}_{1}{ }^{+}$-Gruppe die 1. Satelliten in der $\mathrm{C}_{4}{ }^{++}$. Gruppe korreliert, wo als Repräsentanten nur die $\mathrm{C}_{4} \mathrm{H}_{3}{ }^{++}$-Ionen gefunden werden konnten. Daneben wird man als Ergänzung zu den $\mathrm{C}_{3}{ }^{++}$-Satellitionen entsprechend energiereiche Ionen in der $\mathrm{C}_{2}{ }^{+}$-Gruppe erwarten. Solche Gruppen zeichnen sich bei den Massenzahlen 28 und 29 mehr oder weniger deutlich durch „Knicke“ in den Ausläufern der 1. Satellitverteilungen ab. Wie Tab. 4 zeigt, stimmen die Impulse dieser Ionengruppen recht gut mit denen der Satellitionen in der $\mathrm{C}_{3}{ }^{++}$-Gruppe überein ${ }^{15}$.

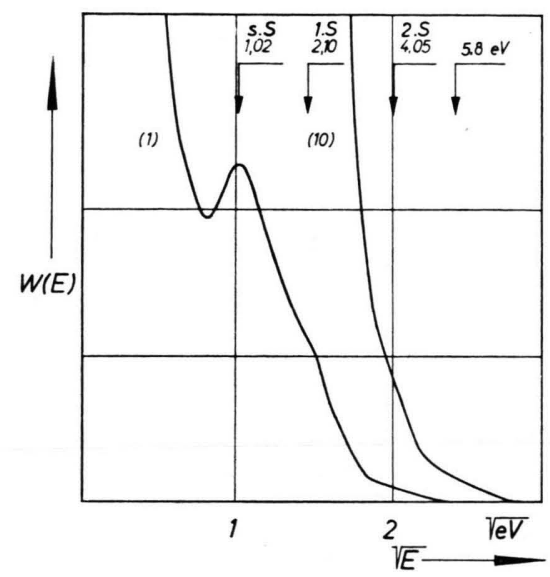

Abb. 8. AE-Verteilung $W(E)$ der $\mathrm{C}_{2} \mathrm{H}_{4}{ }^{+}$-Ionen im n-Pentan oberhalb $0,3 \mathrm{eV}$, aufgetragen über $V E$. Sekundärer, 1. und 2. Satellit sind nach Lage und Energie durch die ersten 3 Pfeile gekennzeichnet. Der Pfeil bei $5,8 \mathrm{eV}$ soll auf die im Text erwähnte weitere Ionengruppe hinweisen. Die Zahlen an den

Kurvenzügen geben die gegenseitige Überhöhung an.

In der Verteilungskurve der Massenzahl 28 von nPentan deutet sich neben der 2. Satellitgruppe durch einen weiteren "Knick“ eine Ionengruppe an, die sich zu noch höheren Energien hin erstreckt (Abb. 8). Beim Versuch, diese Gruppe zu deuten, muß auch die Möglichkeit einer Verunreinigung durch $\mathrm{CO}_{2}$ mit in $\mathrm{Be}$ tracht gezogen werden, da der in $\mathrm{V}^{16}$ untersuchte Prozeß

$$
\mathrm{CO}_{2}^{++} \rightarrow \mathrm{CO}^{+}+\mathrm{O}^{+}
$$

gerade $\mathrm{CO}^{+}$-Ionen der passenden Energie $(5,5 \mathrm{eV})$ liefert. Eine grobe Abschätzung zeigt jedoch, daß dann der $\mathrm{CO}_{2}$-Anteil rund $0,5 \%$ bis $1 \%$ betragen müßte, was sowohl in bezug auf eine Verunreinigung des verwendeten n-Pentans durch $\mathrm{CO}_{2}$ (Lösung) als auch in bezug auf eine mögliche $\mathrm{CO}_{2}$-Bildung durch eine Reaktion am Heizfaden zu hoch erscheint, zumal weder beim i-Pentan noch bei den anderen Kohlenwasserstoffen eine ähnliche Gruppe bei der Massenzahl 28 festgestellt werden

15 Beim Neopentan wurde nach solchen 2. Satelliten in der $\mathrm{C}_{2}{ }^{+}$-Gruppe nicht gesucht. konnte. Apparative Effekte scheiden damit ebenfalls aus, so daß es sich mit großer Wahrscheinlichkeit beim n-Pentan um eine neue Satellitgruppe unter den $\mathrm{C}_{2} \mathrm{H}_{4}{ }^{+}$. Ionen handelt. Ihre Entstehung könnte z. B. durch einen Kaskadenzerfall der Form

$$
\begin{aligned}
\mathrm{C}_{5}^{+++} & \rightarrow \mathrm{C}_{3}{ }^{++}+\mathrm{C}_{2}^{+} \\
& \rightarrow \mathrm{C}_{2}^{+}+\mathrm{C}_{1}^{+} \text {(s. Anm. }{ }^{17} \text { ) }
\end{aligned}
$$

erklärt werden, der in einer gewissen Analogie zu den Prozessen steht, die bei doppeltgeladenen Molekülionen zu den bei den Pentanen ebenfalls nur bei der Massenzahl 28 auftretenden „sekundären“ Satelliten (siehe IV) führen. Von der dazugehörigen, sehr weit auseinandergezogenen Energieverteilungskurve würde nach außen hin dann nur der Ausläufer bei hoher Energie in Erscheinung treten. Allerdings können auch andere Prozesse, z. B. ein sofortiger Dreierzerfall des Molekülions oder Prozesse, die von ein- oder zweifachgeladenen Molekülionen ausgehen, nicht ohne weiteres ausgeschlossen werden.

Hexane. Bei den Hexanen sind die 2. Satelliten der $\mathrm{C}_{1}{ }^{+}$. und $\mathrm{C}_{2}{ }^{+}$-Gruppen zu den 1. Satelliten der $\mathrm{C}_{5}{ }^{++}$- bzw. $\mathrm{C}_{4}{ }^{++}$-Gruppen korreliert und die zugehörigen Impulse stimmen jeweils recht gut miteinander überein. Gegenüber den Pentanen treten hier die 2. Satelliten der $\mathrm{C}_{2}{ }^{+}$-Gruppen mit größerer Deutlichkeit und Intensität hervor (siehe auch Tab. 6).

$\mathrm{Zu}$ den Satelliten in den $\mathrm{C}_{3}{ }^{++}$-Gruppen sollten 2. Satelliten in den $\mathrm{C}_{3}{ }^{+}$-Gruppen korreliert sein. Es konnten jedoch bis auf eine einzige Ausnahme keine solchen Gruppen gefunden werden. Berechnet man die Energien derartiger Ionen aus den Satellitenergien $\operatorname{der} \mathrm{C}_{3}{ }^{++}$. Gruppen mit Hilfe des Impulssatzes, so stellt man fest, $\mathrm{da} ß$ solche Gruppen bei geringer Intensität durch die Ausläufer der 1. Satelliten überdeckt werden und deshalb unbemerkt bleiben. Die oben erwähnte Ausnahme deutet sich nur bei den $\mathrm{C}_{3} \mathrm{H}_{6}{ }^{+}$-Ionen im $\mathrm{n}$-Hexan gut sichtbar durch einen Wendepunkt im Ausläufer der Verteilung als eine weitere energiereiche Ionengruppe an. Sie hat jedoch gegenüber den Satelliten der $\mathrm{C}_{3}{ }^{++}$. Gruppe eindeutig einen zu großen Impuls. Es ist daher unwahrscheinlich, daß diese Gruppen miteinander korreliert sind. Man könnte zwar, wie bei dem 3. Satelliten in der AE-Verteilung der $\mathrm{C}_{2} \mathrm{H}_{4}{ }^{+}$-Ionen im n-Pentan an einen Doppelzerfall

$$
\begin{aligned}
\mathrm{C}_{6}{ }^{+++} & \rightarrow \mathrm{C}_{5}^{++}+\mathrm{C}_{1}^{+} \\
& \rightarrow \mathrm{C}_{3}^{+}+\mathrm{C}_{2}^{+}
\end{aligned}
$$

denken, da der eine Ausläufer der dazugehörigen sehr breiten Energieverteilung gerade an der richtigen Stelle liegen würde. In einem ähnlichen Fall beim n-Heptan scheidet eine derartige Erklärung jedoch aus. Außerdem spricht auch die Form der beim n-Hexan gemessenen Verteilungskurve, ebenso wie beim n-Heptan, mehr für eine in der Energie enger begrenzte Ionengruppe.

16 R. Fuchs u. R. Taubert, Z. Naturforschg. 20 a, 823 [1965].

17 H-Atome sind der Einfachheit halber weggelassen. 
Ein Doppelzerfall als Ursache für den bei der Massenzahl 42 auftretenden 2. Satelliten muß deshalb auch beim n-Hexan als unwahrscheinlich erachtet werden.

Ein weiterer Aufschluß über die Herkunft der 2. Satellitgruppen sollte sich auch aus Auftrittspotentialmessungen ergeben. Die für die Anfangsenergiemessungen benutzte Ionenquelle ist jedoch wegen der hier anzuwendenden extrem hohen Ziehfelder für Auftrittspotentialmessungen schlecht geeignet. Orientierende Messungen wurden deshalb nur beim 2. Satelliten der $\mathrm{CH}_{3}{ }^{+}$-Ionen im n-Hexan versucht. Es ergab sich für diese Ionen ein Auftrittspotential von $64 \pm 4 \mathrm{~V}$, ein Wert, der auch unter Berücksichtigung der kinetischen Energie für eine dreifache Ionisierung im Hexan noch ausreichend sein dürfte.

$n$-Heptan. Beim Heptan sind den Impulsen nach als einzige nur die 2. Satelliten $\operatorname{der} \mathrm{C}_{2}{ }^{+}$-Gruppe und die 1. Satelliten der $\mathrm{C}_{5}{ }^{++}$-Gruppe zueinander korreliert, während Ionen der $\mathrm{C}_{6}{ }^{++}$-Gruppe, die die Ergänzung zur $\mathrm{C}_{1}{ }^{+}$-Gruppe darstellen sollten, vermutlich wegen des schlechten Massenauflösungsvermögens nicht mehr gefunden werden konnten. Auch in den API-Spektren ${ }^{10}$ sind bei den Heptanen zweifachgeladene Ionen der $\mathrm{C}_{6}$-Gruppe nicht aufgeführt. Es konnten aber auch die zu den 1 . Satelliten der $\mathrm{C}_{3}{ }^{++}$. Gruppe und $\mathrm{C}_{4}{ }^{++}$-Gruppe gehörenden Ionen der $\mathrm{C}_{4}{ }^{+}$. bzw. $\mathrm{C}_{3}{ }^{+}$-Gruppe nicht mit Sicherheit nachgewiesen werden. In beiden Fällen ergab eine Nachprüfung, daß solche Ionengruppen sich in ihren Energien mit den 1. Satelliten überlappen und deshalb bei entsprechend geringer Intensität in den Verteilungen nicht mehr hervortreten.

Die deutlich sichtbare 2. Satellitgruppe bei der Massenzahl 42 (Abb. 2) hat gegenüber den $\mathrm{C}_{4} \mathrm{H}_{3}{ }^{++}$-Ionen einen zu großen Impuls. Wie schon bei der Besprechung des Hexans angedeutet, kann sie (abgesehen davon, $\mathrm{da} ß$ es sich $\mathrm{Abb} .2$ zufolge nicht um eine breit verschmierte Energieverteilung handelt) auch energetisch durch keinen der denkbaren Kaskadenzerfälle eines dreifachgeladenen Heptanions erklärt werden. Es ist wahrscheinlich, daß beim n-Hexan und n-Heptan analoge Prozesse zu diesen 2. Satellitgruppen unter den $\mathrm{C}_{3} \mathrm{H}_{6}{ }^{+}$-Ionen führen; weitere Aussagen darüber können jedoch auf Grund der vorliegenden Messungen nicht gemacht werden.

Im Spektrum des n-Heptans treten nun zum erstenmal auch bei doppeltgeladenen Ionen, nämlich in der $\mathrm{C}_{3}{ }^{++}$-Gruppe (Abb. 3), sehr deutlich 2. Satelliten auf, und zwar bei allen Ionen dieser Gruppe. Die Energie dieser 2. Satelliten ist wieder rund doppelt so groß wie die der ersten. In analoger Übertragung der für die 2. Satelliten der $\mathrm{C}^{+}$-Gruppen postulierten Entstehungshypothese ist man geneigt, hier den Zerfall eines vierfachgeladenen Molekülions in zwei doppeltgeladene Teile zugrunde zu legen.

Bis auf einige Ausnahmen ist also angenäherte Impulsgleichheit für korrelierte C-Gruppen vorhanden. Es wäre merkwürdig, wenn diese Erfüllung des Impulssatzes in allen Fällen nur durch Zufall vorgetäuscht wäre. Es ist deshalb anzunehmen, daß bei der Bildung dieser einfach- und doppeltgeladenen Ionen hoher Anfangsenergie überwiegend ladungstrennende Prozesse, die von dreifachgeladenen Molekülionen ausgehen, eine Rolle spielen. Doch können daneben auch andere Prozesse - darauf deuten möglicherweise die 2. Satelliten bei den $\mathrm{C}_{3} \mathrm{H}_{6}{ }^{+}$-Ionen in Hexan und Heptan hin - beteiligt sein. In den meisten Fällen dürfte ihr Beitrag jedoch so gering sein, daß dadurch die Anfangsenergieverteilung der auf die erste Art und Weise gebildeten Ionen durch Úberlagerung nur wenig verändert wird. Die verschiedenen Prozesse lassen sich $u$. U. durch genaue Auftrittspotentialmessungen voneinander unterscheiden ${ }^{14}$.

\section{Ladungsabstände}

Aus den Energien korrelierter Satelliten lassen sich effektive Ladungsabstände $r_{\text {eff }}$ errechnen, d. h. solche Abstände, wie sie die mit den auseinanderlaufenden Teilchen verknüpften Ladungsschwerpunkte im Molekülion haben würden, wenn deren kinetische Energie ausschließlich von der Coulomb-Abstoßung herrühren würde. Man hat es mit drei Ladungen zu tun und es ist anzunehmen, $\mathrm{da} \beta$ diese sich nach der Ionisierung im Molekül so zu verteilen suchen, daß ein möglichst günstiger energetischer Zustand erreicht wird. Es wird also zwischen der nach der Trennung mit dem einen Bruchstück verknüpften einfachen Ladung und den anderen beiden dann zum Rest des Moleküls gehörenden Ladungen zwei verschiedene Abstände $r_{1}$ und $r_{2}$ geben. Die potentielle Energie der Coulomb-Abstoßung, die für die Übergangsenergie ${ }^{\ddot{ }} E^{1}, \mathrm{~d}$. h. für die gesamte, auf die beiden Teilchen übertragene kinetische Energie maßgebend ist - von einer Drehung des doppeltgeladenen Bruchstückes nach der Trennung und einer möglichen Ladungsverschiebung darin soll hier einmal abgesehen werden - , wird durch

$$
E_{\mathrm{Pot}}=\operatorname{const}\left(\frac{e^{2}}{r_{1}}+\frac{e^{2}}{r_{2}}\right)
$$


wiedergegeben. Nach außenhin tritt von den beiden Ladungsabständen über die kinetische Energie wegen

$$
\text { ü } E \cong E_{\text {Pot }}=\text { const } \frac{2 e^{2}}{\bar{r}},
$$

wobei die beiden Ladungen in einem Punkt vereinigt gedacht sind, nur ein Mittelwert (harmonisches Mittel)

in Erscheinung.

$$
\bar{r}=\frac{2 r_{1} r_{2}}{\left(r_{1}+r_{2}\right)}
$$

In Tab. 5 sind die nach

$$
\bar{r}_{\text {eff }}=\frac{2 \cdot 14,4}{\ddot{u} E}\left(\bar{r}_{\text {eff }} \text { in } \AA ; \ddot{u ̈} E \text { in } \mathrm{eV}\right)
$$

berechneten mittleren effektiven Ladungsabstände für die verschiedenen Zerfälle dreifachgeladener Molekülionen aufgeführt. Ein Vergleich mit den in der letzten Spalte aufgeführten Werten $\bar{r}_{\text {ber }}$, die unter der Annahme gestreckter Moleküle und einer ener-

\begin{tabular}{|l|c|c|c|}
\hline \multirow{2}{*}{\multicolumn{1}{|c|}{ Substanz }} & \multicolumn{2}{|c|}{$\bar{r}_{\text {eff }}(\AA)$} & \multirow{2}{*}{$\bar{r}_{\text {ber }}(\AA)$} \\
\cline { 2 - 3 } & \multicolumn{2}{|c|}{ Korrelation } \\
& $\mathrm{C}_{1}^{+} / \mathrm{C}_{n-1}^{++}$ & $\mathrm{C}_{2}^{+} / \mathrm{C}_{n-2}^{++}$ & \\
\hline n-Butan & 3,83 & & 3,72 \\
i-Butan & 3,67 & & 2,87 \\
n-Pentan & 4,24 & 4,20 & 4,61 \\
i-Pentan & 4,13 & 4,05 & 3,72 \\
neo-Pentan & 3,90 & & 2,87 \\
n-Hexan & 4,70 & 4,54 & 5,40 \\
2 Methyl-Pentan & 4,50 & 4,46 & 4,61 \\
2,3-Dimethyl-Butan & 4,28 & & 3,72 \\
n-Heptan & & 4,87 & 6,29 \\
\hline
\end{tabular}

Tab. 5. Mittlere effektive Ladungsabstände $\overline{\boldsymbol{r}}_{\mathrm{eff}}$, berechnet nach der Beziehung (7). ü $E$ ist dabei die Summe der in je zwei korrelierten C-Gruppen ungewichtet gemittelten Energien aus den Tab. 1 und 3. $n=$ Zahl der C-Atome in den undissoziierten Molekülen. In der letzten Spalte der Tabelle sind die für gestreckte n-Paraffine nach der Beziehung (6) berechneten mittleren Ladungsabstände $\bar{r}_{\text {ber }}$ aufgeführt. Dabei wurde angenommen, daß sich die drei Ladungen im Molekülion vor dem Zerfall an den beiden äußeren Wasserstoffatomen und in der Mitte dazwischen befinden.

getisch möglichst günstigen Verteilung der drei Ladungen darin berechnet wurden (s. Tabellenunterschrift), zeigt, daß die aus den Messungen abgeleiteten Ladungsabstände die richtige Größenordnung haben. Aus dieser Tabelle lassen sich nun praktisch die gleichen Gesetzmäßigkeiten ablesen wie aus IV Tab. $2^{1}$, doch sind die Unterschiede der Ladungsabstände für die Korrelationen $\mathrm{C}_{1}{ }^{+} / \mathrm{C}_{n-1}{ }^{++}$und

18 Beim Vergleich von Tab. 5 mit IV Tab. 2 ist wieder die unterschiedliche Kalibrierung der Energieskala zu beachten (siehe Abschn. 1).
$\mathrm{C}_{2}{ }^{+} / \mathrm{C}_{n-2}{ }^{++}$geringer als dort. Die effektiven mittleren Ladungsabstände für dreifachgeladene Molekülionen sind, wie den Tabellen zu entnehmen ist, zwar im allgemeinen etwas kleiner als die für zweifachgeladene ${ }^{18}$, jedoch nicht in dem Maße, wie man unter der Annahme einer energetisch möglichst günstigen Ladungsverteilung vor dem Auseinanderbrechen des Molekülions erwarten würde. Man muß aber berücksichtigen, daß es sich bei den hier benutzten Bildern um grobe Vereinfachungen handelt, die z. B. auch die zweifellos vorhandenen Austauschkräfte außer acht lassen. Aus einem Vergleich der in Tab. 5 und IV, Tab. 2, aufgeführten effektiven Ladungsabstände lassen sich deshalb auch keine Schlüsse hinsichtlich des Unterschiedes in der Verteilung der Ladungsschwerpunkte bei den verschiedenen Zerfallsprozessen ziehen.

In Abb. 9 sind für die Paraffine die allein aus den Energien der 1. und 2. Satelliten der $\mathrm{CH}_{3}{ }^{+}$-Ionen unter der Voraussetzung der Gültigkeit des Impulssatzes und der Annahme, daß beim Zerfall der Molekülionen nur zwei Bruchstücke gebildet werden, berechneten effektiven Ladungsabstände $\bar{r}_{\text {eff }}{ }^{*}$ über der Kohlenstoffzahl der Moleküle aufgetragen. Bei den n-Paraffinen liegen sowohl die zu den 1. als auch die zu den 2. Satelliten gehörenden Ladungsabstände jeweils recht gut auf einer glatten Kurve, die für die 1. Satelliten gekrümmt, für die 2. Satelliten jedoch eine Gerade ist.

Diesen Unterschied in der Kurvenform kann man sich folgendermaßen plausibel machen: Bei den Molekülen der n-Paraffine werden infolge der Drehbarkeit um die Kohlenstoffvalenzen gekrümmte Strukturen eine mit wachsender Kettenlänge zunehmende Rolle spielen ${ }^{19}$, d. h. der Abstand zwischen den am weitesten voneinander entfernten Atomen in solchen Molekülen bleibt im Mittel immer mehr hinter der Länge der ausgestreckten Kette zurück. Nach der in IV ${ }^{1}$ entwickelten Vorstellung wird bei einer zu Ionen hoher Anfangsenergie führenden Zweifachionisation das Elektronenpaar einer (hier äußeren) C-C-Bindung durch ein ionisierendes Elektron entfernt und die beiden dadurch gebildeien Ladungsschwerpunkte nehmen durch Umordnung der Elektronenhülle so rasch einen möglichst großen Abstand voneinander ein, $\mathrm{da} ß$ sich in dieser Zeit die Bruchstücke praktisch noch nicht bewegt haben. Zur kinetischen Energie der Bruchstücke trägt dann nur noch die zu diesem vergrößerten Ladungsabstand gehörige Сочцомв-Energie bei, während der Rest sich in der Anregungsenergie der beiden Ionen wiederfinden

19 Siehe z. B.: H. A. Stuart, Die Struktur des freien Moleküls, Springer-Verlag, Berlin 1952, S. 241. 


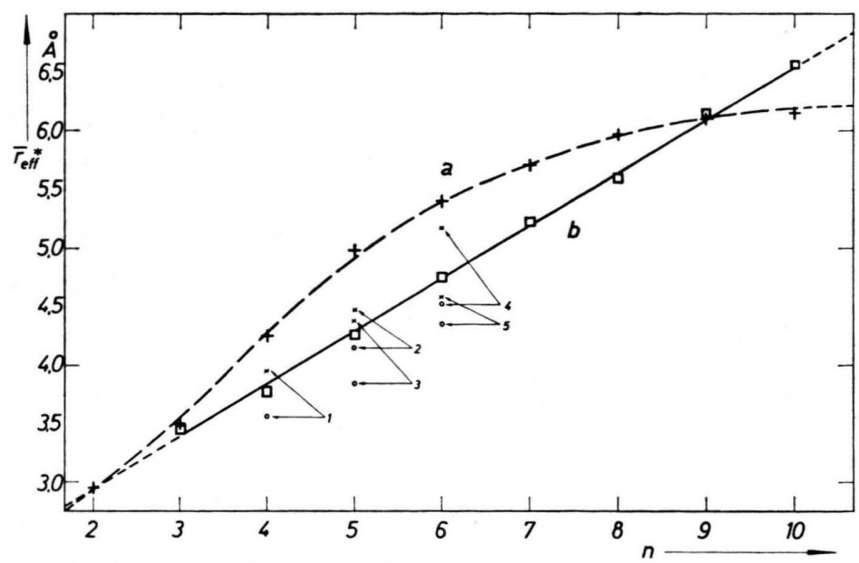

Abb. 9. Mittlere effektive Ladungsabstände $\bar{r}$ eff ${ }^{*}$ für die Zerfälle

$\begin{array}{ll}\mathrm{C}_{n}^{++} \rightarrow \mathrm{C}_{1}^{+}+\mathrm{C}_{n-1}{ }^{+} & \text {(a) } \\ \text { und } \quad \mathrm{C}_{n}^{+++} \rightarrow \mathrm{C}_{1}^{+}+\mathrm{C}_{n-1}++ & \text { (b) }\end{array}$

bei den Paraffinen, errechnet aus den 1. und 2. Satellitenergien der $\mathrm{CH}_{3}{ }^{+}$-Ionen, aufgetragen über der Kohlenstoffzahl $n$ der Moleküle. Große Kreuze und Quadrate gehören zu den n-Paraffinen, kleine Kreuze und Kreise zu 1) i-Butan, 2) i-Pentan, 3) neoPentan, 4) 2-Methyl-Pentan und 5) 2,3-Dimethyl-Butan. muß. Die Zunahme gekrümmter Strukturen bei den Molekülen mit wachsender Kettenlänge spiegelt sich dann in der konkaven Krümmung der Kurve für die effektiven Ladungsabstände wieder.

Bei einer dreifachen Ionisation wird man nun im Mittel die gleichen Molekülstrukturen voraussetzen können wie bei einer Zweifachionisierung. Da es um die Abspaltung von $\mathrm{CH}_{3}{ }^{+}$-Ionen geht, sei wie oben weiterhin angenommen, daß von den drei aus dem Molekül zu entfernenden Elektronen zwei zu einer der äußeren $\mathrm{C}$-C-Bindungen gehören, das Molekül also an dieser Stelle auseinanderbricht.

Die drei Ladungsschwerpunkte streben dann, ähnlich wie im Fall der Zweifachionisation, möglichst weit auseinander. Während des Auseinanderfliegens der Molekülbruchstücke werden auf den größeren Rest, falls er eine gekrümmte Struktur besitzt, durch die beiden mit ihm verknüpften Ladungen streckende Kräfte ausgeübt. Unter der Annahme, daß diese "Streckung“ schon in der ersten Phase des Auseinanderfliegens erfolgt, kann der lineare Anstieg von $\bar{r}_{\text {eff }}{ }^{*}$ mit der C-Zahl gegenüber dem gekrümmten Verlauf dieser Kurve beim Zerfall zweifach ionisierter Moleküle wenigstens qualitativ erklärt werden. Ein Teil der nach der ersten Umordnung der Elektronenhülle noch vorhandenen potentiellen Energie wird sich dabei in inneren Rotationen, in Torsionsschwingungen und schließlich in einer Rotation des gesamten Restes wiederfinden, geht also für die kinetische Energie der Bruchstücke verloren. Zu einer Rotation des dopeltgeladenen Restes nach dem Auseinanderfliegen wird es auch bei gestreckter Anordnung des dreifachgeladenenen Molekülions kommen, da eine solche Anordnung nach dem Bindungsbruch durch Drehung in einen Zustand geringerer potentieller Energie übergehen kann.

\section{Relative Intensitäten}

Wie schon erwähnt, nimmt die Intensität der 2. Satelliten gegenüber der der 1. Satelliten mit wachsender C-Zahl im Molekül zu. Schon aus Tab. 6 ist dieses Anwachsen für die darin aufgeführten Massen- zahlen in der $\mathrm{C}_{2}$-Gruppe zu erkennen. Diese Angaben sind jedoch mehr qualitativ zu werten, da die zur Trennung der beiden Gruppen in den Verteilungskurven nötigen Extrapolationen noch recht will-

\begin{tabular}{|l|c|c|c|c|}
\hline \multirow{2}{*}{ Substanz } & \multicolumn{4}{|c|}{$h_{2} / h_{1}$} \\
\cline { 2 - 5 } & 26 & 27 & 28 & 29 \\
\hline n-Pentan & - & - & $0,07-0,13$ & 0,0029 \\
n-Hexan & - & 0,055 & 0,058 & 0,0045 \\
n-Heptan & 0,094 & 0,059 & 0,070 & 0,0076 \\
n-Nonan & 0,203 & 0,085 & 0,094 & 0,0167 \\
\hline
\end{tabular}

Tab. 6. Verhältnis $h_{2} / h_{1}$ der Höhen der Maxima bei den z. Tl. durch Extrapolation gewonnenen 2. und 1. Satellitverteilungen in den $\mathrm{C}_{2}{ }^{+}$-Gruppen der n-Paraffine.

kürlich sind. Günstiger für eine Trennung der beiden Satellitgruppen liegen die Verhältnisse bei den Anfangsenergieverteilungen der $\mathrm{CH}_{3}{ }^{+}$-Ionen (siehe auch Abb. 1). Aber auch hier geht es nicht ganz ohne Willkür bei der Trennung der beiden Gruppen $a b$ : Mit steigender C-Zahl rücken diese immer weiter zusammen, bis sie schließlich beim n-Tridecan scheinbar nur noch als eine einzige Gruppe wahrzunehmen sind (Abb. 10). Eine Extrapolation wird daher zunehmend unsicherer. In Abb. 11 sind die durch Ausmessen der Flächen unter den extrapolierten Verteilungen gewonnenen Intensitätsverhältnisse für die 2. und 1. Satelliten über der C-Zahl der Moleküle aufgetragen. Die Werte für die n-Paraffine passen wieder gut auf eine glatte Kurve, für die Isomeren scheinen sie im allgemeinen darunter zu liegen. Das starke Anwachsen der 2. Satelliten relativ zu den ersten - von $1-2 \%$ beim n-Butan bis nahe $50 \%$ beim n-Octan und n-Nonan - steht hier außer Zweifel. Ob dagegen die Intensitätsverhältnisse nach Erreichen eines Maximums beim n-Nonan wieder klei- 


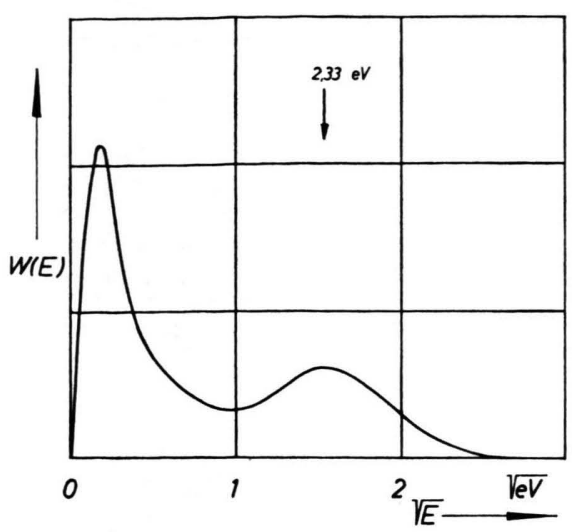

Abb. 10. AE-Verteilung $W(E)$ der $\mathrm{CH}_{3}{ }^{+}$-Ionen in n-Tridecan, aufgetragen über $V E$.

ner werden oder asymptotisch einem Grenzwert zustreben, muß wegen der durch die zunehmende Überlagerung der beiden Gruppen bedingten Unsicherheit bei der Bestimmung ihrer Intensitäten hier offenbleiben.

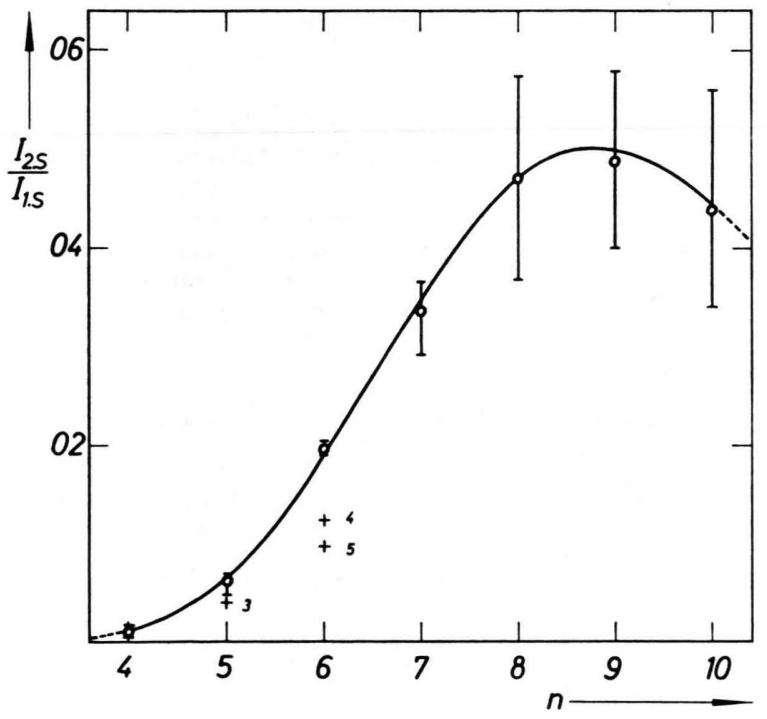

Abb. 11. Verhältnisse der Intensitäten der 2. und 1. Satelliten bei den $\mathrm{CH}_{3}{ }^{+}$-Ionen der Paraffine, aufgetragen über der Kohlenstoffzahl $n$ der Moleküle. Die Kreise gehören zu den nParaffinen, die Kreuze zu 3) neo-Pentan, 4) 2-Methyl-Pentan und 5) 2,3-Dimethyl-Butan. Die für die n-Paraffine eingezeichneten Unsicherheitsgrenzen ergeben sich aus nach beiden Seiten hin extremen Extrapolationen zur Trennung der beiden Gruppen in den AE-Verteilungen. Die eingezeichneten Werte sind die daraus gewonnenen Mittelwerte.

Der Verlauf dieser Kurve kann durch verschiedene Ursachen bedingt sein. Einmal ist es denkbar, da $\beta$ die Elektronenenergie $U_{\mathrm{e}}$ dabei eine Rolle spielt: Wie aus einem Vergleich der Satellitintensitäten der $\mathrm{CH}_{3}{ }^{+}$-Ionen in n-Hexan und n-Nonan bei einem ef- fektiven $U_{\mathrm{e}}$ von 70 und $150 \mathrm{eV}$ zu ersehen ist, scheinen sich die "Ionisierungskurven" (damit sei hier die Intensität der gesamten Gruppe in Abhängigkeit von $U_{\mathrm{e}}$ bezeichnet) mit zunehmender Molekülgröße zu niedrigerem $U_{\mathrm{e}}$ hin zu verschieben. Bei einer fest eingestellten Elektronenenergie mißt man für die verschiedenen Substanzen dann an verschiedenen Stellen der als „ähnlich“ angenommenen „Ionisierungskurven“. Gleichzeitig kommt es für das Intensitätsverhältnis auch noch auf die Form und Lage dieser Kurven bei den 1. Satelliten an. Allerdings waren beim n-Butan und neo-Pentan zwischen $U_{\mathrm{e}}$ $=150 \mathrm{eV}$ und $250 \mathrm{eV}$ bzw. $350 \mathrm{eV}$ keine merklichen Änderungen der Satellitintensitäten mehr zu finden. Bei $U_{\mathrm{e}}=150 \mathrm{eV}$ scheint man also bei diesen Substanzen schon oberhalb des Anstiegs der „Ionisierungskurven" zu liegen, so daß allein damit das oben erwähnte starke Anwachsen des Satellitintensitätsverhältnisses nicht erklärt werden kann, möglicherweise aber der angedeutete Abfall nach größeren Molekülen hin. Mehr darüber sollte aus detaillierteren Messungen der Abhängigkeit der Satellitintensitäten von der Elektronenenergie zu erfahren sein.

Eine andere Erklärungsmöglichkeit für den Anstieg der Kurve geht von der hier vertretenen Modellvorstellung, die den Zerfall eines mehrfachgeladenen Molekülions in geladene Bruchstücke als Ursache der in dieser Arbeit besprochenen Ionen hoher Anfangsenergie voraussetzt, aus: Zwei von den drei bei der primären Ionisation aus dem Molekül entfernten Elektronen stammen aus einer der äußeren $\mathrm{C}$-C-Bindungen, während das dritte auch von einer beliebigen anderen Stelle kommen kann. Dann ist die Wahrscheinlichkeit, daß außer der zweifachen Ionisation bei der C - C-Bindung noch eine weitere erfolgt, um so größer, je ausgedehnter das Molekül ist, d. h. je länger das ionisierende Elektron mit dessen Elektronenhülle in Wechselwirkung treten kann ${ }^{20}$. Die Struktur der Moleküle (mehr oder weniger stark gekrümmte Formen bei den n-Paraffinen, Verzweigungen bei den Isomeren) mag dabei ebenfalls von Einfluß sein.

Herrn Prof. Dr. TAubert danke ich für kritische Diskussionen. Der Deutschen Forschungsgemeinschaft habe ich für die Überlassung des verwendeten Massenspektrometers zu danken.

20 Unterstützt wird diese Vorstellung auch von der Tatsache, daß sich beim Äthan in der Anfangsenergie-Verteilung der $\mathrm{CH}_{3}{ }^{+}$-Ionen bei Elektronenenergien bis zu $300 \mathrm{eV}$ keinerlei Andeutung eines 2. Satelliten zeigte. 